\title{
Cuntz-Krieger-Pimsner Algebras Associated with Amalgamated Free Product Groups
}

\author{
By
}

Rui OKAYASU*

\begin{abstract}
We give a construction of a nuclear $C^{*}$-algebra associated with an amalgamated free product of groups, generalizing Spielberg's construction of a certain CuntzKrieger algebra associated with a finitely generated free product of cyclic groups. Our nuclear $C^{*}$-algebras can be identified with certain Cuntz-Krieger-Pimsner algebras. We will also show that our algebras can be obtained by the crossed product construction of the canonical actions on the hyperbolic boundaries, which proves a special case of Adams' result about amenability of the boundary action for hyperbolic groups. We will also give an explicit formula of the $K$-groups of our algebras. Finally we will investigate a relationship between the KMS states of the generalized gauge actions on our $C^{*}$ algebras and random walks on the groups.
\end{abstract}

\section{$\S 1$ Introduction}

In [5], Choi proved that the reduced group $C^{*}$-algebra $C_{r}^{*}\left(\mathbb{Z}_{2} * \mathbb{Z}_{3}\right)$ of the free product of cyclic groups $\mathbb{Z}_{2}$ and $\mathbb{Z}_{3}$ is embedded in $\mathcal{O}_{2}$. Consequently, this shows that $C_{r}^{*}\left(\mathbb{Z}_{2} * \mathbb{Z}_{3}\right)$ is a non-nuclear exact $C^{*}$-algebra, (see S. Wassermann [31] for a good introduction to exact $C^{*}$-algebras). Spielberg generalized it to finitely generated free products of cyclic groups in [28]. Namely, he constructed a certain action on a compact space and proved that some Cuntz-Krieger algebras (see [8]) can be obtained by the crossed product construction for the action. For a related topic, see W. Szymański and S. Zhang's work [30].

Communicated by Y. Takanashi. Received October 3, 2000.

2000 Mathematics Subject Classification(s): 46L05.

* Department of Mathematics, Kyoto University, Kyoto 606-8502, Japan.

e-mail: rui@kusm.kyoto-u.ac.jp 
More generally, the above mentioned compact space coincides with Gromov's notion of the boundaries of hyperbolic groups (e.g. see [18]). In [1], Adams proved that the action of any discrete hyperbolic group $\Gamma$ on the hyperbolic boundary $\partial \Gamma$ is amenable in the sense of Anantharaman-Delaroche [2]. It follows from [2] that the corresponding crossed product $C(\partial \Gamma) \rtimes_{r} \Gamma$ is nuclear, and this implies that $C_{r}^{*}(\Gamma)$ is an exact $C^{*}$-algebra.

Although we know that $C(\partial \Gamma) \rtimes_{r} \Gamma$ is nuclear for a general discrete hyperbolic group $\Gamma$ as mentioned above, there are only few things known about this $C^{*}$-algebra. So one of our purposes is to generalize Spielberg's construction to some finitely generated amalgamated free product $\Gamma$ and to give detailed description of the algebra $C(\partial \Gamma) \rtimes_{r} \Gamma$. More precisely, let $I$ be a finite index set and $G_{i}$ be a group containing a copy of a finite group $H$ as a subgroup for $i \in I$. We always assume that each $G_{i}$ is either a finite group or $\mathbb{Z} \times H$. Let $\Gamma={ }^{H} G_{i}$ be the amalgamated free product group. We will construct a nuclear $C^{*}$-algebra $\mathcal{O}_{\Gamma}$ associated with $\Gamma$ by mimicking the construction for Cuntz-Krieger algebras with respect to the full Fock space in M. Enomoto, M. Fujii and Y. Watatani [12] and D. E. Evans [14]. This generalizes Spielberg's construction.

First we show that $\mathcal{O}_{\Gamma}$ has a certain universal property as in the case of the Cuntz-Krieger algebras, which allows several descriptions of $\mathcal{O}_{\Gamma}$. For example, it turns out that $\mathcal{O}_{\Gamma}$ is a Cuntz-Krieger-Pimsner algebra, introduced by Pimsner in [23] and studied by several authors, e.g. T. Kajiwara, C. Pinzari and Y. Watatani [19]. We will also show that $\mathcal{O}_{\Gamma}$ can be obtained by the crossed product construction. Namely, we will introduce a boundary space $\Omega$ with a natural $\Gamma$-action, which coincides with the boundary of the associated tree (see [27], [32]). Then we will prove that $C(\Omega) \rtimes_{r} \Gamma$ is isomorphic to $\mathcal{O}_{\Gamma}$. Since the hyperbolic boundary $\partial \Gamma$ coincides with $\Omega$ and the two actions of $\Gamma$ on $\partial \Gamma$ and $\Omega$ are conjugate, $\mathcal{O}_{\Gamma}$ is also isomorphic to $C(\partial \Gamma) \rtimes_{r} \Gamma$, and depends only on the group structure of $\Gamma$. As a consequence, we give a proof to Adams' theorem in this special case.

Next, we will consider the $K$-groups of $\mathcal{O}_{\Gamma}$. In [22], Pimsner gave a certain exact sequence of $K K$-groups of the crossed product by groups acting on trees. However, it is not a trivial task to apply Pimsner's exact sequence to $C(\partial \Gamma) \rtimes_{r} \Gamma$ and obtain its $K$-groups. We will give explicit formulae of the $K$-groups of $\mathcal{O}_{\Gamma}$ following the method used for the Cuntz-Krieger algebras instead of using $C(\partial \Gamma) \rtimes_{r} \Gamma$. We can compute the $K$-groups of $C(\partial \Gamma) \rtimes_{r} \Gamma$ for concrete examples. They are completely determined by the representation theory of $H$ and the actions of $H$ on $G_{i} / H$ (the space of right cosets) by left multiplication. 
Finally we will prove that KMS states on $\mathcal{O}_{\Gamma}$ for generalized gauge actions arise from harmonic measures on the Poisson boundary with respect to random walks on the discrete group $\Gamma$. Consequently, for special cases, we can determine easily the type of factor $\mathcal{O}_{\Gamma}^{\prime \prime}$ for the corresponding unique KMS state of the gauge action by essentially the same arguments in M. Enomoto, M. Fujii and Y. Watatani [13], which generalized J. Ramagge and G. Robertson's result [25].

\section{$\S 2 . \quad$ Preliminaries}

In this section, we collect basic facts used in the present article. We begin by reviewing the Cuntz-Krieger-Pimsner algebras in [23]. Let $A$ be a $C^{*}$-algebra and $X$ be a Hilbert bimodule over $A$, which means that $X$ is a right Hilbert $A$-module with an injective $*$-homomorphism of $A$ to $\mathcal{L}(X)$, where $\mathcal{L}(X)$ is the $C^{*}$-algebra of all adjointable $A$-linear operators on $X$. We assume that $X$ is full, that is, $\left\{\langle x, y\rangle_{A} \mid x, y \in X\right\}$ generates $A$ as a $C^{*}$-algebra, where $\langle\cdot, \cdot\rangle_{A}$ is the $A$-valued inner product on $X$. We further assume that $X$ has a finite basis $\left\{u_{1}, \ldots, u_{n}\right\}$, which means that $x=\sum_{i=1}^{n} u_{i}\left\langle u_{i}, x\right\rangle_{A}$ for any $x \in X$. We fix a basis $\left\{u_{1}, \ldots, u_{n}\right\}$ of $X$. Let $\mathcal{F}(X)=A \oplus \bigoplus_{n \geq 1} X^{(n)}$ be the full Fock space over $X$, where $X^{(n)}$ is the $n$-fold tensor product $X \otimes_{A} X \otimes_{A} \cdots \otimes_{A} X$. Note that $\mathcal{F}(X)$ is naturally equipped with Hilbert $A$-bimodule structure. For each $x \in X$, the operator $T_{x}: \mathcal{F}(X) \rightarrow \mathcal{F}(X)$ is defined by

$$
\begin{aligned}
T_{x}\left(x_{1} \otimes \cdots \otimes x_{n}\right) & =x \otimes x_{1} \otimes \cdots \otimes x_{n}, \\
T_{x}(a) & =x a,
\end{aligned}
$$

for $x, x_{1}, \ldots, x_{n} \in X$ and $a \in A$. Note that $T_{x} \in \mathcal{L}(\mathcal{F}(X))$ satisfies the following relations

$$
\begin{aligned}
& T_{x}^{*} T_{y}=\langle x, y\rangle_{A}, \quad x, y \in X, \\
& a T_{x} b=T_{a x b}, \quad x \in X, a, b \in A .
\end{aligned}
$$

Let $\pi$ be the quotient map of $\mathcal{L}(\mathcal{F}(X))$ onto $\mathcal{L}(\mathcal{F}(X)) / \mathcal{K}(\mathcal{F}(X))$ where $\mathcal{K}(\mathcal{F}(X))$ is the $C^{*}$-algebra of all compact operators of $\mathcal{L}(\mathcal{F}(X))$. We denote $S_{x}=\pi\left(T_{x}\right)$ for $x \in X$. Then we define the Cuntz-Krieger-Pimsner algebra $\mathcal{O}_{X}$ to be

$$
\mathcal{O}_{X}=C^{*}\left(S_{x} \mid x \in X\right)
$$

Since $X$ is full, a copy of $A$ acting by left multiplication on $\mathcal{F}(X)$ is contained in $\mathcal{O}_{X}$. Furthermore we have the relation

$$
\sum_{i=1}^{n} S_{u_{i}} S_{u_{i}}^{*}=1 .
$$


On the other hand, $\mathcal{O}_{X}$ is characterized as the universal $C^{*}$-algebra generated by $A$ and $S_{x}$, satisfying the above relations [23, Theorem 3.12]. More precisely, we have

Theorem 2.1 ([23, Theorem 3.12]). $\quad$ Let $X$ be a full Hilbert A-bimodule and $\mathcal{O}_{X}$ be the corresponding Cuntz-Krieger-Pimsner algebra. Suppose that $\left\{u_{1}, \ldots, u_{n}\right\}$ is a finite basis for $X$. If $B$ is a $C^{*}$-algebra generated by $\left\{s_{x}\right\}_{x \in X}$ satisfying

$$
\begin{aligned}
s_{x}+s_{y} & =s_{x+y}, \quad x \in X, \\
a s_{x} b & =s_{a x b}, \quad x \in X, a, b \in A, \\
s_{x}^{*} s_{y} & =\langle x, y\rangle_{A}, \quad x, y \in X, \\
\sum_{i=1}^{n} s_{u_{i}} s_{u_{i}}^{*} & =1 .
\end{aligned}
$$

Then there exists a unique surjective $*$-homomorphism from $\mathcal{O}_{X}$ onto $C^{*}\left(s_{x}\right)$ that maps $S_{x}$ to $s_{x}$.

Next we recall the notion of amenability for discrete $C^{*}$-dynamical systems introduced by C. Anantharaman-Delaroche in [2]. Let $(A, G, \alpha)$ be a $C^{*}$-dynamical system, where $A$ is a $C^{*}$-algebra, $G$ is a group and $\alpha$ is an action of $G$ on $A$. An $A$-valued function $h$ on $G$ is said to be of positive type if the matrix $\left[\alpha_{s_{i}}\left(h\left(s_{i}^{-1} s_{j}\right)\right)\right] \in M_{n}(A)$ is positive for any $s_{1}, \ldots, s_{n} \in G$. We assume that $G$ is discrete. Then $\alpha$ is said to be amenable if there exists a net $\left(h_{i}\right)_{i \in I} \subset C_{c}\left(G, Z\left(A^{\prime \prime}\right)\right)$ of functions of positive type such that

$$
\begin{cases}h_{i}(e) \leq 1 & \text { for } i \in I, \\ \lim _{i} h_{i}(s)=1 & \text { for } s \in G,\end{cases}
$$

where the limit is taken in the $\sigma$-weak topology in the enveloping von Neumann algebra $A^{\prime \prime}$ of $A$. We remark that this is one of several equivalent conditions given in [2, Théorème 3.3$]$. We will use the following theorems without a proof.

Theorem 2.2 ([2, Théorème 4.5]). Let $(A, G, \alpha)$ be a $C^{*}$-dynamical system such that $A$ is nuclear and $G$ is discrete. Then the following are equivalent:

1) The full $C^{*}$-crossed product $A \rtimes_{\alpha} G$ is nuclear;

2) The reduced $C^{*}$-crossed product $A \rtimes_{\alpha r} G$ is nuclear;

3) The $W^{*}$-crossed product $A^{\prime \prime} \rtimes_{\alpha w} G$ is injective;

4) The action $\alpha$ of $G$ on $A$ is amenable. 
Theorem 2.3 ([2, Théorème 4.8]). Let $(A, G, \alpha)$ be an amenable $C^{*}$ dynamical system such that $G$ is discrete. Then the natural quotient map from $A \rtimes_{\alpha} G$ onto $A \rtimes_{\alpha r} G$ is an isomorphism.

Finally, we review the notion of the strong boundary actions in [21]. Let $\Gamma$ be a discrete group acting by homeomorphisms on a compact Hausdorff space $\Omega$. Suppose that $\Omega$ has at least three points. The action of $\Gamma$ on $\Omega$ is said to be a strong boundary action if for every pair $U, V$ of non-empty open subsets of $\Omega$ there exists $\gamma \in \Gamma$ such that $\gamma U^{c} \subset V$. The action of $\Gamma$ on $\Omega$ is said to be topologically free in the sense of [3] if the fixed point set of each non-trivial element of $\Gamma$ has empty interior.

Theorem 2.4 ([21, Theorem 5$])$. Let $(\Omega, \Gamma)$ be a strong boundary action where $\Omega$ is compact. We further assume that the action is topologically free. Then $C(\Omega) \rtimes_{r} \Gamma$ is purely infinite and simple.

\section{§3. A Motivating Example}

Before introducing our algebras, we present a simple case of Spielberg's construction for $\mathbb{F}_{2}=\mathbb{Z} * \mathbb{Z}$ with generators $a$ and $b$ as a motivating example. See also [26]. The Cayley graph of $\mathbb{F}_{2}$ is a homogeneous tree of degree 4 . The boundary $\Omega$ of the tree in the sense of [16] (see also [17]) can be thought of as the set of all infinite reduced words $\omega=x_{1} x_{2} x_{3} \cdots$, where $x_{i} \in S=\left\{a, b, a^{-1}, b^{-1}\right\}$. Note that $\Omega$ is compact in the relative topology of the product topology of $\prod_{\mathbb{N}} S$. In an appendix, several facts about trees are collected for the convenience of the reader, (see also [15]). Left multiplication of $\mathbb{F}_{2}$ on $\Omega$ induces an action of $\mathbb{F}_{2}$ on $C(\Omega)$. For $x \in \mathbb{F}_{2}$, let $\Omega(x)$ be the set of infinite words beginning with $x$. We identify the implementing unitaries in the full crossed product $C(\Omega) \rtimes \mathbb{F}_{2}$ with elements of $\mathbb{F}_{2}$. Let $p_{x}$ denote the projection defined by the characteristic function $\chi_{\Omega(x)} \in C(\Omega)$. Note that for each $x \in S$,

$$
\begin{aligned}
p_{x}+x p_{x^{-1}} x^{-1} & =1, \\
p_{a}+p_{a^{-1}}+p_{b}+p_{b^{-1}} & =1,
\end{aligned}
$$

hold. For $x \in S$, let $S_{x} \in C(\Omega) \rtimes \mathbb{F}_{2}$ be a partial isometry

$$
S_{x}=x\left(1-p_{x^{-1}}\right) .
$$

Then we have

$$
S_{x}^{*} S_{y}=x^{-1} p_{x} p_{y} y=\delta_{x, y} S_{x}^{*} S_{x}=\delta_{x, y}\left(1-p_{x^{-1}}\right),
$$




$$
\begin{gathered}
S_{x} S_{x}^{*}=x\left(1-p_{x^{-1}}\right) x^{-1}=p_{x}, \\
S_{x}^{*} S_{x}=1-p_{x^{-1}}=\sum_{y \neq x^{-1}} S_{y} S_{y}^{*} .
\end{gathered}
$$

These relations show that the partial isometries $S_{x}$ generate the Cuntz-Krieger algebra $\mathcal{O}_{A}[8]$, where

$$
A=\left(\begin{array}{llll}
1 & 0 & 1 & 1 \\
0 & 1 & 1 & 1 \\
1 & 1 & 1 & 0 \\
1 & 1 & 0 & 1
\end{array}\right)
$$

On the other hand, we can recover the generators of $C(\Omega) \rtimes \mathbb{F}_{2}$ by setting

$$
x=S_{x}+S_{x^{-1}}^{*} \quad \text { and } \quad p_{x}=S_{x} S_{x}^{*} .
$$

Hence we have $C(\Omega) \rtimes \mathbb{F}_{2} \simeq \mathcal{O}_{A}$.

Next we recall the Fock space realization of the Cuntz-Krieger algebras, (e.g. see [14], [12]). Let $\left\{e_{a}, e_{b}, e_{a^{-1}}, e_{b^{-1}}\right\}$ be a basis of $\mathbb{C}^{4}$. We define the Fock space associated with the matrix $A$ by

$$
\mathcal{F}_{A}=\mathbb{C} e_{0} \oplus \bigoplus_{n \geq 1}\left(\overline{\operatorname{span}}\left\{e_{x_{1}} \otimes \cdots \otimes e_{x_{n}} \mid A\left(x_{i}, x_{i+1}\right)=1\right\}\right)
$$

where $e_{0}$ is the vacuum vector. For any $x \in S$, let $T_{x}$ be the creation operator on $\mathcal{F}$, given by

$$
\begin{aligned}
T_{x} e_{0} & =e_{x}, \\
T_{x}\left(e_{x_{1}} \otimes \cdots \otimes e_{x_{n}}\right) & = \begin{cases}e_{x} \otimes e_{x_{1}} \otimes \cdots \otimes e_{x_{n}} & \text { if } A\left(x, x_{1}\right)=1, \\
0 & \text { otherwise. }\end{cases}
\end{aligned}
$$

Let $p_{0}$ be the rank one projection on the vacuum vector $e_{0}$. Note that we have

$$
T_{a} T_{a}^{*}+T_{b} T_{b}^{*}+T_{a^{-1}} T_{a^{-1}}^{*}+T_{b^{-1}} T_{b^{-1}}^{*}+p_{0}=1 .
$$

If $\pi$ is the quotient map of $\mathcal{B}(\mathcal{F})$ onto the Calkin algebra $\mathcal{Q}(\mathcal{F})$, then the $C^{*}$ algebra generated by the partial isometries $\left\{\pi\left(T_{a}\right), \pi\left(T_{b}\right), \pi\left(T_{a^{-1}}\right), \pi\left(T_{b^{-1}}\right)\right\}$ is isomorphic to the Cuntz-Krieger algebra $\mathcal{O}_{A}$.

Now we look at this construction from another point of view. We can perform the following natural identification:

$$
\mathcal{F} \ni \underset{e_{x_{1}} \otimes \cdots \otimes e_{x_{n}} \longleftrightarrow \delta_{x_{1} \cdots x_{n}}}{e_{0}} \in l^{2}\left(\mathbb{F}_{2}\right)
$$


Under this identification, the creation operator $T_{x}$ on $l^{2}\left(\mathbb{F}_{2}\right)$ can be expressed as

$$
\begin{aligned}
T_{x} \delta_{e} & =\lambda_{x} \delta_{e} \\
T_{x} \delta_{x_{1} \cdots x_{n}} & = \begin{cases}\lambda_{x} \delta_{x_{1} \cdots x_{n}} & \text { if } x \neq x_{1}^{-1} \\
0 & \text { otherwise }\end{cases}
\end{aligned}
$$

where $\lambda$ is the left regular representation of $\mathbb{F}_{2}$.

For a reduced word $x_{1} \cdots x_{n} \in \mathbb{F}_{2}$, we define the length function $|\cdot|$ on $\mathbb{F}_{2}$ by $\left|x_{1} \cdots x_{n}\right|=n$. Let $p_{n}$ be the projection onto the closed linear span of $\left\{\delta_{\gamma} \in l^{2}\left(\mathbb{F}_{2}\right)|| \gamma \mid=n\right\}$. Then we can express $T_{x}$ for $x \in S$ by

$$
T_{x}=\sum_{n \geq 0} p_{n+1} \lambda_{x} p_{n}
$$

Note that this expression makes sense for every finitely generated group. In the next section, we generalize this construction to amalgamated free product groups.

\section{$\S 4$. Construction of a Nuclear $C^{*}$-algebra $\mathcal{O}_{\Gamma}$}

In what follows, we always assume that $I$ is a finite index set and $G_{i}$ is a group containing a copy of a finite group $H$ as a subgroup for $i \in I$. Moreover, we assume that each $G_{i}$ is either a finite group or $\mathbb{Z} \times H$. We set $I_{0}=\left\{i \in I|| G_{i} \mid<\infty\right\}$. Let $\Gamma=*_{H} G_{i}$ be the amalgamated free product.

First we introduce a "length function" $|\cdot|$ on each $G_{i}$. If $i \in I_{0}$, we set $|g|=1$ for any $g \in G_{i} \backslash H$ and $|h|=0$ for any $h \in H$. If $i \in I \backslash I_{0}$ we set $\left|\left(a_{i}^{n}, h\right)\right|=|n|$ for any $\left(a_{i}^{n}, h\right) \in G_{i}=\mathbb{Z} \times H$ where $a_{i}$ is a generator of $\mathbb{Z}$. Now we extend the length function to $\Gamma$. Let $\Omega_{i}$ be a set of left representatives of $G_{i} / H$ with $e \in \Omega_{i}$. If $\gamma \in \Gamma$ is written uniquely as $g_{1} \cdots g_{n} h$, where $g_{1} \in$ $\Omega_{i_{1}}, \ldots, g_{n} \in \Omega_{i_{n}}$ with $i_{1} \neq i_{2}, \ldots, i_{n-1} \neq i_{n}$ (we write simply $i_{1} \neq \cdots \neq i_{n}$ ), then we define

$$
|\gamma|=\sum_{k=1}^{n}\left|g_{k}\right|
$$

Let $p_{n}$ be the projection of $l^{2}(\Gamma)$ onto $l^{2}\left(\Gamma_{n}\right)$ for each $n$, where $\Gamma_{n}=\{\gamma \in$ $\Gamma|| \gamma \mid=n\}$. We define partial isometries and unitary operators on $l^{2}(\Gamma)$ by

$$
\begin{cases}T_{g}=\sum_{n \geq 0} p_{n+1} \lambda_{g} p_{n} & \text { if } g \in \bigcup_{i \in I} G_{i} \backslash H, \\ V_{h}=\lambda_{h} & \text { if } h \in H,\end{cases}
$$

where $\lambda$ is the left regular representation of $\Gamma$. Let $\pi$ be the quotient map of $\mathcal{B}\left(l^{2}(\Gamma)\right)$ onto $\mathcal{B}\left(l^{2}(\Gamma)\right) / \mathcal{K}\left(l^{2}(\Gamma)\right)$, where $\mathcal{B}\left(l^{2}(\Gamma)\right)$ is the $C^{*}$-algebra of all 
bounded linear operators on $l^{2}(\Gamma)$ and $\mathcal{K}\left(l^{2}(\Gamma)\right)$ is the $C^{*}$-subalgebra of all compact operators of $\mathcal{B}\left(l^{2}(\Gamma)\right)$. We set $\pi\left(T_{g}\right)=S_{g}$ and $\pi\left(V_{h}\right)=U_{h}$. For $\gamma \in \Gamma$, we define $S_{\gamma}$ by

$$
S_{\gamma}=S_{g_{1}} \cdots S_{g_{n}},
$$

where $\gamma=g_{1} \cdots g_{n}$ for some $g_{1} \in G_{i_{1}} \backslash H, \ldots, g_{n} \in G_{i_{n}} \backslash H$ with $i_{1} \neq \cdots \neq i_{n}$. Note that $S_{\gamma}$ does not depend on the expression $\gamma=g_{1} \cdots g_{n}$. We denote the initial projections of $S_{\gamma}$ by $Q_{\gamma}=S_{\gamma}^{*} \cdot S_{\gamma}$ and the range projections by $P_{\gamma}=S_{\gamma} \cdot S_{\gamma}^{*}$ for $\gamma \in \Gamma$.

We collect several relations, which the family $\left\{S_{g}, U_{h} \mid g \in \bigcup_{i \in I} G_{i} \backslash H, h \in\right.$ $H\}$ satisfies.

For $g, g^{\prime} \in \bigcup_{i} G_{i} \backslash H$ with $|g|=\left|g^{\prime}\right|=1$ and $h \in H$,

$$
\begin{gathered}
S_{g h}=S_{g} \cdot U_{h}, \quad S_{h g}=U_{h} \cdot S_{g}, \\
P_{g} \cdot P_{g^{\prime}}= \begin{cases}P_{g}=P_{g^{\prime}} & \text { if } g H=g^{\prime} H, \\
0 & \text { if } g H \neq g^{\prime} H .\end{cases}
\end{gathered}
$$

Moreover, if $g \in G_{i} \backslash H$ and $i \in I_{0}$, then

$$
Q_{g}=\sum_{\substack{j \in I_{0} \\ j \neq i}} \sum_{g^{\prime} \in \Omega_{j} \backslash\{e\}} P_{g^{\prime}}+\sum_{j \in I \backslash I_{0}} P_{a_{j}}+P_{a_{j}^{-1}},
$$

and if $g=a_{i}^{ \pm 1}$ and $i \in I \backslash I_{0}$, then

$$
Q_{a_{i}^{ \pm 1}}=\sum_{j \in I_{0}} \sum_{g^{\prime} \in \Omega_{j} \backslash\{e\}} P_{g^{\prime}}+\sum_{\substack{j \in I \backslash I_{0} \\ j \neq i}}\left(P_{a_{j}}+P_{a_{j}^{-1}}\right)+P_{a_{i}^{ \pm 1}}
$$

Finally,

$$
1=\sum_{i \in I_{0}} \sum_{g \in \Omega_{i} \backslash\{e\}} P_{g}+\sum_{i \in I \backslash I_{0}}\left(P_{a_{i}}+P_{a_{i}^{-1}}\right) .
$$

Indeed, (1) follows from the relations $T_{g h}=T_{g} V_{h}$ and $T_{h g}=V_{h} T_{g}$. From the definition, we have $T_{g^{\prime}}^{*} T_{g}=\sum_{n \geq 0} p_{n} \lambda_{g^{\prime}}^{*} p_{n+1} \lambda_{g} p_{n}$. This can be non-zero if and only if $\left|g^{\prime-1} g\right|=0$, i.e. $g^{\prime-1} g \in H$. We have (2) immediately. The relation

$$
1=\sum_{i \in I_{0}} \sum_{g \in \Omega_{i}} T_{g} T_{g}^{*}+\sum_{i \in I \backslash I_{0}}\left(T_{a_{i}} T_{a_{i}}^{*}+T_{a_{i}^{-1}} T_{a_{i}^{-1}}^{*}\right)+p_{0},
$$

implies (4). By multiplying $S_{g}^{*}$ on the left and $S_{g}$ on the right of equation (4) respectively, we obtain (3). 
Moreover, the following condition holds: Let $P_{i}=\sum_{g \in \Omega_{i}} P_{g}$ for $i \in I_{0}$, and $P_{i}=P_{a_{i}}+P_{a_{i}^{-1}}$ for $i \in I \backslash I_{0}$. For every $i \in I$, we have

$$
C^{*}(H) \simeq C^{*}\left(P_{i} U_{h} P_{i} \mid h \in H\right) .
$$

Indeed, since the unitary representation $P_{i}^{\prime} V_{h} P_{i}^{\prime}$ contains the left regular representation of $H$ with infinite multiplicity, where $P_{i}^{\prime}$ is some projection with $\pi\left(P_{i}^{\prime}\right)=P_{i}$. we have relation $(5)$.

Now we consider the universal $C^{*}$-algebra generated by the family $\left\{S_{g}, U_{h} \mid\right.$ $\left.g \in \bigcup_{i \in I} G_{i} \backslash H, h \in H\right\}$ satisfying (1), (2), (3) and (4). We denote it by $\mathcal{O}_{\Gamma}$. Here, the universality means that if another family $\left\{s_{g}, u_{h}\right\}$ satisfies $(1),(2),(3)$ and (4), then there exists a surjective $*$-homomorphism $\phi$ of $\mathcal{O}_{\Gamma}$ onto $C^{*}\left(s_{g}, u_{h}\right)$ such that $\phi\left(S_{g}\right)=s_{g}$ and $\phi\left(U_{h}\right)=u_{h}$. Summing up the above, we employ the following definitions and notation:

Definition 4.1. Let $I$ be a finite index set and $G_{i}$ be a group containing a copy of a finite group $H$ as a subgroup for $i \in I$. Suppose that each $G_{i}$ is either a finite group or $\mathbb{Z} \times H$. Let $I_{0}$ be the subset of $I$ such that $G_{i}$ is finite for all $i \in I_{0}$. We denote the amalgamated free product $*_{H} G_{i}$ by $\Gamma$.

We fix a set $\Omega_{i}$ of left representatives of $G_{i} / H$ with $e \in \Omega_{i}$ and a set $X_{i}$ of representatives of $H \backslash G_{i} / H$ which is contained in $\Omega_{i}$. Let $\left(a_{i}, e\right)$ be a generator of $G_{i}$ for $i \in I \backslash I_{0}$. We write $a_{i}$, for short. Here we choose $\Omega_{i}=X_{i}=\left\{a_{i}^{n} \mid n \in \mathbb{N}\right\}$. We exclude the case where $\bigcup_{i} \Omega_{i} \backslash\{e\}$ has only one or two points.

We define the corresponding universal $C^{*}$-algebra $\mathcal{O}_{\Gamma}$ generated by partial isometries $S_{g}$ for $g \in \bigcup_{i \in I} G_{i} \backslash H$ and unitaries $U_{h}$ for $h \in H$ satisfying (1), (2), (3) and (4).

We set for $\gamma \in \Gamma$,

$$
\begin{aligned}
Q_{\gamma} & =S_{\gamma}^{*} \cdot S_{\gamma}, \quad P_{\gamma}=S_{\gamma} \cdot S_{\gamma}^{*}, \\
P_{i} & =\sum_{g \in \Omega_{i}} P_{g} \quad \text { if } i \in I_{0}, \\
P_{i} & =P_{a_{i}}+P_{a_{i}^{-1}} \quad \text { if } i \in I \backslash I_{0} .
\end{aligned}
$$

For convenience, we set for any integer $n$,

$$
\begin{aligned}
\Gamma_{n} & =\{\gamma \in \Gamma|| \gamma \mid=n\}, \\
\Delta_{n} & =\left\{\gamma \in \Gamma_{n} \mid \gamma=\gamma_{1} \cdots \gamma_{n}, \gamma_{k} \in \Omega_{i_{k}}, i_{1} \neq \cdots \neq i_{n}\right\} .
\end{aligned}
$$

We also set $\Delta=\bigcup_{n \geq 1} \Delta_{n}$. 
Lemma 4.2. For $i \in I$ and $h \in H$,

$$
U_{h} P_{i}=P_{i} U_{h}
$$

Proof. Use the above relations (2).

Lemma 4.3. Let $\gamma_{1}, \gamma_{2} \in \Gamma$. Suppose that $S_{\gamma_{1}}^{*} S_{\gamma_{2}} \neq 0$.

If $\left|\gamma_{1}\right|=\left|\gamma_{2}\right|$, then $S_{\gamma_{1}}^{*} S_{\gamma_{2}}=Q_{g} U_{h}$ for some $g \in \bigcup_{i \in I} G_{i}, h \in H$.

If $\left|\gamma_{1}\right|>\left|\gamma_{2}\right|$, then $S_{\gamma_{1}}^{*} S_{\gamma_{2}}=S_{\gamma}^{*}$ for some $\gamma \in \Gamma$ with $|\gamma|=\left|\gamma_{1}\right|-\left|\gamma_{2}\right|$.

If $\left|\gamma_{1}\right|<\left|\gamma_{2}\right|$, then $S_{\gamma_{1}}^{*} S_{\gamma_{2}}=S_{\gamma}$ for some $\gamma \in \Gamma$ with $|\gamma|=\left|\gamma_{2}\right|-\left|\gamma_{1}\right|$.

Proof. By (2), we obtain the lemma.

\section{Corollary 4.4.}

$$
\mathcal{O}_{\Gamma}=\overline{\operatorname{span}}\left\{S_{\mu} P_{i} S_{\nu}^{*} \mid \mu, \nu \in \Gamma, i \in I\right\}
$$

Proof. This follows from the previous lemma.

Next we consider the gauge action of $\mathcal{O}_{\Gamma}$. Namely, if $z \in \mathbb{T}$ then the family $\left\{z S_{g}, U_{h}\right\}$ also satisfies (1), (2), (3), (4) and generates $\mathcal{O}_{\Gamma}$. The universality gives an automorphism $\alpha_{z}$ on $\mathcal{O}_{\Gamma}$ such that $\alpha_{z}\left(S_{g}\right)=z S_{g}$ and $\alpha_{z}\left(U_{h}\right)=U_{h}$. In fact, $\alpha$ is a continuous action of $\mathbb{T}$ on $\mathcal{O}_{\Gamma}$, which is called the gauge action. Let $d z$ be the normalized Haar measure on $\mathbb{T}$ and we define a conditional expectation $\Phi$ of $\mathcal{O}_{\Gamma}$ onto the fixed-point algebra $\mathcal{O}_{\Gamma}^{\mathbb{T}}=\left\{a \in \mathcal{O}_{\Gamma} \mid \alpha_{z}(a)=a\right.$, for $\left.z \in \mathbb{T}\right\}$ by

$$
\Phi(a)=\int_{\mathbb{T}} \alpha_{z}(a) d z, \quad \text { for } a \in \mathcal{O}_{\Gamma} .
$$

Lemma 4.5. The fixed-point algebra $\mathcal{O}_{\Gamma}^{\mathbb{T}}$ is an AF-algebra.

Proof. For each $i \in I$, set

$$
\mathcal{F}_{n}^{i}=\overline{\operatorname{span}}\left\{S_{\mu} P_{i} S_{\nu}^{*} \mid \mu, \nu \in \Gamma_{n}\right\} .
$$

We can find systems of matrix units in $\mathcal{F}_{n}^{i}$, parameterized by $\mu, \nu \in \Delta_{n}$, as follows:

$$
e_{\mu, \nu}^{i}=S_{\mu} P_{i} S_{\nu}^{*} .
$$

Indeed, using the previous lemma, we compute

$$
e_{\mu_{1}, \nu_{1}}^{i} e_{\mu_{2}, \nu_{2}}^{i}=\delta_{\nu_{1}, \mu_{2}} S_{\mu_{1}} P_{i} Q_{\nu_{1}} P_{i} S_{\nu_{2}}^{*}=\delta_{\nu_{1}, \mu_{2}} e_{\mu_{1}, \nu_{2}}^{i} .
$$


Thus we obtain the identifications

$$
\mathcal{F}_{n}^{i} \simeq M_{N(n, i)}(\mathbb{C}) \otimes e_{\mu, \mu}^{i} \mathcal{F}_{n}^{i} e_{\mu, \mu}^{i},
$$

for some integer $N(n, i)$ and some $\mu \in \Delta_{n}$. Moreover, for $\xi, \eta$,

$$
e_{\mu, \mu}^{i}\left(S_{\xi} P_{i} S_{\eta}^{*}\right) e_{\mu, \mu}^{i}= \begin{cases}S_{\mu} P_{i} U_{h} P_{i} S_{\mu}^{*} & \text { if } \xi, \eta \in \mu H \\ 0 & \text { otherwise. }\end{cases}
$$

for some $h \in H$. Note that $C^{*}\left(S_{\mu} P_{i} U_{h} P_{i} S_{\mu}^{*} \mid h \in H\right)$ is isomorphic to $C^{*}\left(P_{i} U_{h} P_{i} \mid h \in H\right)$ via the map $x \mapsto S_{\mu}^{*} x S_{\mu}$. Therefore the relation (5) gives

$$
\mathcal{F}_{n}^{i} \simeq M_{k}(\mathbb{C}) \otimes \overline{\operatorname{span}}\left\{S_{\mu} P_{i} U_{h} P_{i} S_{\mu}^{*} \mid h \in H\right\} \simeq M_{k}(\mathbb{C}) \otimes C^{*}(H) .
$$

Note that $\left\{\mathcal{F}_{n}^{i} \mid i \in I\right\}$ are mutually orthogonal and

$$
\mathcal{F}_{n}=\bigoplus_{i \in I} \mathcal{F}_{n}^{i}
$$

is a finite-dimensional $C^{*}$-algebra.

The relation (2) gives $\mathcal{F}_{n} \hookrightarrow \mathcal{F}_{n+1}$. Hence,

$$
\mathcal{F}=\overline{\bigcup_{n \geq 0} \mathcal{F}_{n}}
$$

is an $A F$-algebra. Therefore it suffices to show that $\mathcal{F}=\mathcal{O}_{\Gamma}^{\mathbb{T}}$. It is trivial that $\mathcal{F} \subseteq \mathcal{O}_{\Gamma}^{\mathbb{T}}$. On the other hand, we can approximate any $a \in \mathcal{O}_{\Gamma}^{\mathbb{T}}$ by a linear combination of elements of the form $S_{\mu} P_{i} S_{\nu}^{*}$. Since $\Phi(a)=a, a$ can be approximated by a linear combination of elements of the form $S_{\mu} P_{i} S_{\nu}^{*}$ with $|\mu|=|\nu|$. Thus $a \in \mathcal{F}$.

We need another lemma to prove the uniqueness of $\mathcal{O}_{\Gamma}$.

Lemma 4.6. $\quad$ Suppose that $i_{0} \in I$ and $W$ consists of finitely many elements $(\mu, h) \in \Delta \times H$ such that the last word of $\mu$ is not contained in $\Omega_{i_{0}}$ and $W \cap\{e\} \times H=\emptyset$. Then there exists $\gamma=g_{0} \cdots g_{n}$ with $g_{k} \in \Omega_{i_{k}}$ and $i_{0} \neq \cdots \neq i_{n} \neq i_{0}$ such that for any $(\mu, h) \in W, \mu h \gamma$ never have the form $\gamma \gamma^{\prime}$ for some $\gamma^{\prime} \in \Gamma$.

Proof. Let $i_{0} \in I$ and $W$ be a finite subset of $\Delta \times H$ as above. We first assume that $|I| \geq 3$. Then we can choose $x \in \Omega_{i_{0}}, y \in \Omega_{j}$ and $z \in \Omega_{j^{\prime}}$ such that $j \neq i_{0} \neq j^{\prime}$ and $j \neq j^{\prime}$. For sufficiently long word

$$
\gamma=(x y)(x z)(x y x y)(x z x z)(x y x y x y)(x z x z x z) \cdots(\cdots z),
$$


we are done. We next assume that $|I|=2$. Since we exclude the case where $\Omega_{1} \cup \Omega_{2} \backslash\{e\}$ has only one or two elements, we can choose at least three distinct points $x \in \Omega_{i_{0}}, y \in \Omega_{j}$ and $z \in \Omega_{j^{\prime}}$. If $i_{0} \neq j=j^{\prime}$ we set

$$
\gamma=(x y)(x z)(x y x y)(x z x z)(x y x y x y)(x z x z x z) \cdots(\cdots z),
$$

as well. If $i_{0}=j \neq j^{\prime}$ we set

$$
\gamma=(x z)(y z)(x z x z)(y z y z)(x z x z x z)(y z y z y z) \cdots(\cdots z) .
$$

Then if $\gamma$ has the desired properties, we are done. Now assume that there exist some $(\mu, h) \in W$ such that $\mu h \gamma=\gamma \gamma^{\prime}$ for some $\gamma^{\prime}$. Fix such an element $(\mu, h) \in W$. By hypothesis, we can choose $\delta \in \Delta$ with $\left|\gamma^{\prime}\right| \leq|\delta|$ such that the last word of $\delta$ does not belong to $\Omega_{i_{0}}$ and $\delta$ does not have the form $\gamma^{\prime} \delta^{\prime}$ for some $\delta^{\prime}$. Set $\tilde{\gamma}=\gamma \delta$. Then $\mu h \tilde{\gamma}$ does not have the form $\gamma \gamma^{\prime \prime}$ for any $\gamma^{\prime \prime}$. Indeed,

$$
\mu h \tilde{\gamma}=\mu h \gamma \delta=\gamma \gamma^{\prime} \delta \neq \tilde{\gamma} \gamma^{\prime \prime}
$$

for some $\gamma^{\prime \prime}$. Since $W$ is finite, we can obtain a desired element $\gamma$ by replacing $\tilde{\gamma}$, inductively.

We now obtain the uniqueness theorem for $\mathcal{O}_{\Gamma}$.

Theorem 4.7. Let $\left\{s_{g}, u_{h}\right\}$ be another family of partial isometries and unitaries satisfying (1), (2), (3) and (4). Assume that

$$
C^{*}(H) \simeq C^{*}\left(p_{i} u_{h} p_{i} \mid h \in H\right),
$$

where $p_{i}=\sum_{g \in \Omega_{i} \backslash\{e\}} s_{g} s_{g}^{*}$ for $i \in I_{0}$ and $p_{i}=s_{a_{i}} s_{a_{i}}^{*}+s_{a_{i}^{-1}} s_{a_{i}^{-1}}^{*}$ for $i \in I \backslash I_{0}$. Then the canonical surjective *-homomorphism $\pi$ of $\mathcal{O}_{\Gamma}$ onto $C^{*}\left(s_{g}, u_{h}\right)$ is faithful.

Proof. To prove the theorem, it is enough to show that (a) $\pi$ is faithful on the fixed-point algebra $\mathcal{O}_{\Gamma}^{\mathbb{T}}$, and (b) $\|\pi(\Phi(a))\| \leq\|\pi(a)\|$ for all $a \in \mathcal{O}_{\Gamma}$ thanks to [4, Lemma 2.2].

To establish (a), it suffices to show that $\pi$ is faithful on $\mathcal{F}_{n}$ for all $n \geq 0$. By the proof of Lemma 4.5, we have

$$
\mathcal{F}_{n}^{i}=M_{N(n, i)}(\mathbb{C}) \otimes C^{*}(H),
$$

for some integer $N(n, i)$. Note that $s_{g} s_{g}^{*}$ is non-zero. Hence $\pi$ is injective on $M_{N(n, i)}(\mathbb{C})$. By the other hypothesis, $\pi$ is injective on $C^{*}(H)$. 
Next we will show (b). It is enough to check (b) for

$$
a=\sum_{\mu, \nu \in F} \sum_{j \in J} C_{\mu, \nu}^{j} S_{\mu} P_{j} S_{\nu}^{*}
$$

where $F$ is a finite subset of $\Gamma$ and $J$ is a subset of $I$. For $n=\max \{|\mu| \mid \mu \in F\}$, we have

$$
\Phi(a)=\sum_{\{\mu, \nu \in F|| \mu|=| \nu \mid\}} \sum_{j \in J} C_{\mu, \nu}^{j} S_{\mu} P_{j} S_{\nu}^{*} \in \mathcal{F}_{n} .
$$

Now by changing $F$ if necessary, we may assume that $\min \{|\mu|,|\nu|\}=n$ for every pair $\mu, \nu \in F$ with $C_{\mu, \nu}^{j} \neq 0$. Since $\mathcal{F}_{n}=\oplus_{i} \mathcal{F}_{n}^{i}$, there exists some $i_{0} \in J$ such that

$$
\|\pi(\Phi(a))\|=\left\|\sum_{|\mu|=|\nu|} C_{\mu, \nu}^{i_{0}} s_{\mu} p_{\imath_{0}} s_{\nu}^{*}\right\| .
$$

By changing $F$ such that $F \subset \Delta$ again, we may further assume that

$$
\|\pi(\Phi(a))\|=\left\|\sum_{\substack{\mu, \nu \in F \\|\mu|=|\nu|}} \sum_{h \in F^{\prime}} C_{\mu, \nu, h}^{i_{0}} s_{\mu} p_{i_{0}} u_{h} p_{i_{0}} s_{\nu}^{*}\right\|
$$

where $F^{\prime}$ consists of elements of $H$, (perhaps with multiplicity). By applying the preceding lemma to

$$
W=\left\{\left(\mu^{\prime}, h\right) \in \Delta \times H \mid \mu^{\prime} \text { is subword of } \mu \in F, h^{-1} \in F^{\prime}\right\},
$$

we have $\gamma \in \Delta$ satisfying the property in the previous lemma. Then we define a projection

$$
Q=\sum_{\tau \in \Delta_{n}} s_{\tau} s_{\gamma} p_{i_{0}} s_{\gamma}^{*} s_{\tau}^{*}
$$

By hypothesis, $Q$ is non-zero.

If $\mu, \nu \in \Delta_{n}$ then

$$
Q\left(s_{\mu} p_{i_{0}} s_{\nu}^{*}\right) Q=s_{\mu} s_{\gamma} p_{i_{0}} s_{\gamma}^{*} p_{i_{0}} s_{\gamma} p_{i_{0}} s_{\gamma}^{*} s_{\nu}^{*}=s_{\mu} s_{\gamma} p_{i_{0}} s_{\gamma}^{*} s_{\nu}^{*}
$$

is non-zero. Therefore $s_{\mu}\left(s_{\gamma} p_{i_{0}} s_{\gamma}^{*}\right) s_{\nu}^{*}$ is also a family of matrix units parameterized by $\mu, \nu \in \Delta_{n}$. Hence the same arguments as in the proof of Lemma 4.5 give

$$
\pi\left(\mathcal{F}_{n}^{i_{0}}\right) \simeq M_{N\left(n, i_{0}\right)}(\mathbb{C}) \otimes C^{*}\left(s_{\mu} s_{\gamma} p_{i_{0}} u_{h} p_{i_{0}} s_{\gamma}^{*} s_{\mu}^{*} \mid h \in H\right) .
$$

By hypothesis, we deduce that $b \mapsto Q \pi(b) Q$ is faithful on $\mathcal{F}_{n}^{i_{0}}$. In particular, we conclude that $\|\pi(\Phi(a))\|=\|Q \pi(\Phi(a)) Q\|$. 
We next claim that $Q \pi(\Phi(a)) Q=Q \pi(a) Q$. We fix $\mu, \nu \in F$. If $|\mu| \neq|\nu|$ then one of $\mu, \nu$ has length $n$ and the other is longer; say $|\mu|=n$ and $|\nu|>n$. Then

$$
Q\left(s_{\mu} p_{i_{0}} u_{h} p_{i_{0}} s_{\nu}^{*}\right) Q=s_{\mu} s_{\gamma} p_{i_{0}} s_{\gamma}^{*} p_{i_{0}} u_{h} p_{i_{0}} s_{\nu}^{*}\left(\sum_{\tau \in \Delta_{n}} s_{\tau} s_{\gamma} p_{i_{0}} s_{\gamma}^{*} s_{\tau}^{*}\right) .
$$

Since $|\nu|>|\tau|$, this can have a non-zero summand only if $\nu=\tau \nu^{\prime}$ for some $\nu^{\prime}$. However $s_{\gamma}^{*} u_{h} s_{\nu}^{*} s_{\tau} s_{\gamma}=s_{\gamma}^{*} u_{h} s_{\nu^{\prime}}^{*} s_{\gamma}$, and $s_{\nu^{\prime} h^{-1} \gamma}^{*} s_{\gamma}$ is non-zero only if $\nu^{\prime} h^{-1} \gamma$ has the form $\gamma \gamma^{\prime}$. This is impossible by the choice of $\gamma$. Therefore we have $Q\left(s_{\mu} p_{i_{0}} s_{\nu}\right) Q=0$ if $|\mu| \neq|\nu|$, namely $Q \pi(\Phi(a)) Q=Q \pi(a) Q$. Hence we can finish proving (b):

$$
\|\pi(\Phi(a))\|=\|Q \pi(\Phi(a)) Q\|=\|Q \pi(a) Q\| \leq\|\pi(a)\| .
$$

Therefore [4, Lemma 2.2] gives the theorem.

By essentially the same arguments, we can prove the following.

Corollary 4.8. Let $\left\{t_{g}, v_{h}\right\}$ and $\left\{s_{g}, u_{h}\right\}$ be two families of partial isometries and unitaries satisfying (1), (2), (3) and (4). Suppose that the map $p_{i} v_{h} p_{i} \mapsto q_{i} u_{h} q_{i}$ gives an isomorphism:

$$
C^{*}\left(p_{i} v_{h} p_{i} \mid h \in H\right) \simeq C^{*}\left(q_{i} v_{h} q_{i} \mid h \in H\right),
$$

where $p_{i}=\sum_{g \in \Omega_{i} \backslash\{e\}} t_{g} t_{g}^{*}, q_{i}=\sum_{g \in \Omega_{i} \backslash\{e\}} s_{g} s_{g}^{*}$ and so on. Then the canonical map gives the isomorphism between $C^{*}\left(t_{g}, v_{h}\right)$ and $C^{*}\left(s_{g}, u_{h}\right)$.

Before closing this section, we will show that our algebra $\mathcal{O}_{\Gamma}$ is isomorphic to a certain Cuntz-Krieger-Pimsner algebra. Let $A=C^{*}\left(P_{i} U_{h} P_{i} \mid h \in H, i \in I\right)$ $\simeq \bigoplus_{i \in I} C_{r}^{*}(H)$. We define a Hilbert $A$-bimodule $X$ as follows:

$$
X=\overline{\operatorname{span}}\left\{S_{g} P_{i}\left|g \in \bigcup_{j \neq i} G_{j},\right| g \mid=1, i \in I\right\}
$$

with respect to the inner product $\left\langle S_{g} P_{i}, S_{g^{\prime}} P_{j}\right\rangle=P_{i} S_{g}^{*} S_{g^{\prime}} P_{j} \in A$. In terms of the groups, the $A$ - $A$ bimodule structure can be described as follows: we set

$$
A=\bigoplus_{i \in I} A_{i}=\bigoplus_{i \in I} \mathbb{C}[H]
$$

and define an $A$-bimodule $\mathcal{H}_{i}$ by

$$
\mathcal{H}_{i}=\mathbb{C}\left[\left\{g \in \bigcup_{j \neq i} G_{j}|| g \mid=1\right\}\right]
$$


with left and right $A$-multiplications such that for $a=\left(h_{i}\right)_{i \in I} \in A$ and $g \in$ $G_{j} \backslash H \subset \mathcal{H}_{i}$

$$
a \cdot g=h_{j} g \text { and } g \cdot a=g h_{i},
$$

and with respect to the inner product

$$
\left\langle g, g^{\prime}\right\rangle_{\mathcal{H}_{i}}= \begin{cases}g^{-1} g^{\prime} \in A_{i} & \text { if } g^{-1} g^{\prime} \in H \\ 0 & \text { otherwise }\end{cases}
$$

Then we define the $A$-bimodule $X$ by

$$
X=\bigoplus_{i \in I} \mathcal{H}_{i}
$$

and we obtain the CKP-algebra $\mathcal{O}_{X}$.

Proposition 4.9. Assume that $A$ and $X$ are as above. Then

$$
\mathcal{O}_{\Gamma} \simeq \mathcal{O}_{X}
$$

Proof. We fix a finite basis $u(g, i)=g \in \mathcal{H}_{i}$ for $g \in \Omega_{j}, i \in I$ with $j \neq i,|g|=1$. Then we have $\mathcal{O}_{X}=C^{*}\left(S_{u(g, i)}\right)$. Let $s_{u(g, i)}=S_{g} P_{i}$ in $\mathcal{O}_{\Gamma}$. Note that we have $\mathcal{O}_{\Gamma}=C^{*}\left(s_{u(g, i)}\right)$. The relation (4) corresponds to the relations $(\dagger)$ of the CKP-algebras. The family $\left\{s_{u(g, i)}\right\}$ therefore satisfies the relations of the CKP-algebras. Since the CKP-algebra has universal properties, there exists a canonical surjective $*$-homomorphism of $\mathcal{O}_{X}$ onto $\mathcal{O}_{\Gamma}$. Conversely, let $s_{g}=\sum_{i \in I} S_{u(g, i)}$ and $u_{h}=\oplus_{i \in I} h$ for $h \in H$ in $\mathcal{O}_{X}$, and then we have $\mathcal{O}_{X}=C^{*}\left(s_{g}, u_{h}\right)$. By the universality of $\mathcal{O}_{\Gamma}$, we can also obtain a canonical surjective $*$-homomorphism of $\mathcal{O}_{\Gamma}$ onto $\mathcal{O}_{X}$. These maps are mutual inverses. Indeed,

$$
\begin{aligned}
S_{g} & \mapsto \sum_{i \in I} S_{u(g, i)} \mapsto \sum_{i \in I} S_{g} P_{i}=S_{g}, \\
U_{h} \mapsto \bigoplus_{i \in I} h & \mapsto \sum_{i \in I} P_{i} U_{h} P_{i}=U_{h} .
\end{aligned}
$$

\section{$\S 5$. Crossed Product Algebras Associated with $\mathcal{O}_{\Gamma}$}

In this section, we will show that $\mathcal{O}_{\Gamma}$ is isomorphic to a crossed product algebra. We first define a "boundary space". We set

$\tilde{\Lambda}=\left\{\left(\gamma_{n}\right)\left|\gamma_{n} \in \Gamma,\right| \gamma_{n}|+1=| \gamma_{n+1}|,| \gamma_{n}^{-1} \gamma_{n+1} \mid=1\right.$ for a sufficiently large $\left.n \geq 0\right\}$.

We introduce the following equivalence relation $\sim ;\left(\gamma_{n}\right)_{n \geq 0},\left(\gamma_{n}^{\prime}\right)_{n \geq 0} \in \tilde{\Lambda}$ are equivalent if there exists some $k \in \mathbb{Z}$ such that $\gamma_{n} H=\gamma_{n+k}^{\prime} H$ for a sufficiently 
large $n$. Then we define $\Lambda=\tilde{\Lambda} / \sim$. We denote the equivalent class of $\left(\gamma_{n}\right)_{n \geq 0}$ by $\left[\gamma_{n}\right]_{n \geq 0}$.

Before we define an action of $\Gamma$ on $\Lambda$, we construct another space $\Omega$ to introduce a compact space structure, on which $\Gamma$ acts continuously. Let $\Omega$ denote the set of sequences $x: \mathbb{N} \rightarrow \Gamma$ such that

$$
\begin{cases}x(n) \in \Omega_{i_{n}} \backslash\{e\} & \text { for } n \geq 1 \\ x(n) \in\left\{a_{i_{n}}^{ \pm 1}\right\} & \text { if } i_{n} \in I \backslash I_{0} \\ i_{n} \neq i_{n+1} & \text { if } i_{n} \in I_{0} \\ x(n)=x(n+1) & \text { if } i_{n} \in I \backslash I_{0}, i_{n}=i_{n+1}\end{cases}
$$

Note that $\Omega$ is a compact Hausdorff subspace of $\prod_{\mathbb{N}}\left(\bigcup_{i} \Omega_{i} \backslash\{e\}\right)$. We introduce a map $\phi$ between $\Lambda$ and $\Omega$; for $x=(x(n))_{n \geq 1} \in \Omega$, we define a map $\phi(x)=$ $\left[\gamma_{n}\right] \in \Lambda$ by

$$
\begin{aligned}
& \gamma_{0}=e \quad \text { if } n=0, \\
& \gamma_{n}=x(1) \cdots x(n), \quad \text { if } n \geq 1 .
\end{aligned}
$$

Lemma 5.1. The above map $\phi$ is a bijection from $\Lambda$ onto $\Omega$ and hence $\Lambda$ inherits a compact space structure via $\phi$.

Proof. For $x=(x(n)) \neq x^{\prime}=\left(x^{\prime}(n)\right)$, there exists an integer $k$ such that $x(k) \neq x^{\prime}(k)$. If $\phi(x)=\left[\gamma_{n}\right]$ and $\phi\left(x^{\prime}\right)=\left[\gamma_{n}^{\prime}\right]$, then $\gamma_{k} H \neq \gamma_{k}^{\prime} H$. Hence we have injectivity of $\phi$. Next we will show surjectivity. Let $\left[\gamma_{n}\right] \in \Sigma$. We may take a representative $\left(\gamma_{n}\right)$ satisfying $\left|\gamma_{n}\right|=n$. Now we assume that $\gamma_{n}$ is uniquely expressed as $\gamma_{n}=g_{1} \cdots g_{n} h, \gamma_{n+1}=g_{1}^{\prime} \cdots g_{n+1}^{\prime} h^{\prime}$ for $g_{k} \in \Omega_{i_{k}}, g_{k}^{\prime} \in \Omega_{j_{k}}, h, h^{\prime} \in$ $H$. Since $\left|\gamma_{n}^{-1} \gamma_{n+1}\right|=1$, we have

$$
h^{-1} g_{n}^{-1} \cdots g_{1}^{-1} g_{1}^{\prime} \cdots g_{n+1}^{\prime} h^{\prime}=g,
$$

for some $g \notin H$ with $|g|=1$. Inductively, we have $g_{1}=g_{1}^{\prime}, \ldots, g_{n}=g_{n}^{\prime}$. Hence we can assume that $\gamma_{n}=g_{1} \cdots g_{n}$. We set $x(n)=g_{n}$ and get $\phi((x(n)))=$ $\left[\gamma_{n}\right]$

Next we define an action of $\Gamma$ on $\Lambda$. Let $\left[\gamma_{n}\right]_{n \geq 0} \in \Lambda$. For $\gamma \in \Gamma$, define

$$
\gamma \cdot\left[\gamma_{n}\right]_{n \geq 0}=\left[\gamma \gamma_{n}\right]_{n \geq 0} .
$$

We will show that this is a continuous action of $\Gamma$ on $\Lambda$. Let $\left[\gamma_{n}\right],\left[\gamma_{n}^{\prime}\right] \in \Lambda$ such that $\left(\gamma_{n}\right) \sim\left(\gamma_{n}^{\prime}\right)$ and $\gamma \in \Gamma$. Since there exists some integer $k$ such that $\gamma_{n} H=\gamma_{n+k}^{\prime} H$ for sufficiently large integers $n$, we have $\gamma \gamma_{n} H=\gamma \gamma_{n+k}^{\prime} H$. 
Hence this is well-defined. To show that $\gamma$ is continuous, we consider how $\gamma$ acts on $\Omega$ via the map $\phi$. For $g \in \Omega_{i}$ with $|g|=1$ and $x=(x(n))_{n \geq 1} \in \Omega$,

$$
(g \cdot x)(1)= \begin{cases}g & \text { if } i \neq i_{1}, \\ g_{1} & \text { if } i=i_{1}, g x(1) \notin H, i \in I_{0}, \\ & \text { and } g x(1)=g_{1} h_{1}\left(g_{1} \in \Omega_{i_{1}}, h_{1} \in H\right), \\ g & \text { if } i=i_{1}, g x(1) \notin H, i \in I \backslash I_{0}, \\ g_{2} & \text { if } i=i_{1}, g x(1) \in H, i \in I_{0}, \\ & \text { and } g x(1)=h_{1}, h_{1} x(2)=g_{2} h_{2}\left(g_{2} \in \Omega_{i_{2}}, h_{1}, h_{2} \in H\right), \\ x(2) & \text { if } i=i_{1}, g x(1) \in H, i \in I \backslash I_{0},\end{cases}
$$

and for $n>1$,

$$
(g \cdot x)(n)= \begin{cases}x(n-1) & \text { if } i \neq i_{1}, \\ g_{n} & \text { if } i=i_{1}, g x(1) \notin H, \\ & \text { and } h_{n-1} x(n)=g_{n} h_{n}\left(g_{n} \in \Omega_{i_{n}}, h_{n} \in H\right), \\ x(n-1) & \text { if } i=i_{1}, g x(1) \notin H, i \in I \backslash I_{0}, \\ g_{n+1} & \text { if } i=i_{1}, g x(1) \in H, \\ & \text { and } h_{n} x(n+1)=g_{n+1} h_{n+1},\left(g_{n+1} \in \Omega_{i_{n+1}}, h_{n+1} \in H\right), \\ x(n+1) & \text { if } i=i_{1}, g x(1) \in H, i \in I \backslash I_{0} .\end{cases}
$$

For $h \in H$,

$$
(h \cdot x)(n)= \begin{cases}g_{1} & \text { if } n=1 \\ & \text { and } h x(1)=g_{1} h_{1},\left(g_{1} \in \Omega_{i_{1}}, h_{n} \in H\right) \\ g_{n} & \text { if } n>1 \\ & \text { and } h_{n-1} x(n)=g_{n} h_{n},\left(g_{n} \in \Omega_{i_{n}}, h_{n} \in H\right) .\end{cases}
$$

Then one can check easily that the pull-back of any open set of $\Omega$ by $\gamma$ is also an open set of $\Omega$. Thus we have proved that $\gamma$ is a homeomorphism on $\Lambda$. The equations

$$
\left(\gamma \gamma^{\prime}\right)\left[\gamma_{n}\right]=\left[\gamma \gamma^{\prime} \gamma_{n}\right]=\gamma\left(\left[\gamma^{\prime} \gamma_{n}\right]\right)=\gamma \circ \gamma^{\prime}\left[\gamma_{n}\right]
$$

imply associativity.

Therefore we have obtained the following:

Lemma 5.2. The above space $\Omega$ is a compact Hausdorff space and $\Gamma$ acts on $\Omega$ continuously.

The following result is the main theorem of this section. 
Theorem 5.3. Assume that $\Omega$ and the action of $\Gamma$ on $\Omega$ are as above. Then we have the identifications

$$
\mathcal{O}_{\Gamma} \simeq C(\Omega) \rtimes \Gamma \simeq C(\Omega) \rtimes_{r} \Gamma
$$

Proof. We first consider the full crossed product $C(\Omega) \rtimes \Gamma$. Let $Y_{i}=$ $\left\{(x(n)) \mid x(1) \in \Omega_{i}\right\} \subset \Omega$ be clopen sets for $i \in I$. Note that if $i \in I_{0}$, then $Y_{i}$ is the disjoint union of the clopen sets $\left\{g\left(\Omega \backslash Y_{i}\right) \mid g \in \Omega_{i} \backslash\{e\}\right\}$, and if $i \in I \backslash I_{0}$, then $Y_{i}=Y_{i}^{+} \cup Y_{i}^{-}$where $Y_{i}^{ \pm}=\left\{(x(n)) \mid x(1)=a_{i}^{ \pm}\right\}$. Let $p_{i}=\chi_{\Omega \backslash Y_{i}}$ and $p_{i}^{ \pm}=\chi_{Y_{i}^{ \pm}}$. We define $T_{g}=g p_{i}$ for $g \in G_{i} \backslash H$ and $i \in I_{0}$ and $T_{a_{i}^{ \pm 1}}=a_{i}^{ \pm 1}\left(p_{i}+p_{i}^{ \pm}\right)$for $i \in I \backslash I_{0}$. Let $V_{h}=h$ for $h \in H$. Then the family $\left\{T_{g}, V_{h}\right\}$ satisfies the relations (1), (2), (3) and (4). Indeed, we can first check that $h \in H$ commutes with $p_{i}$ and $p_{i}^{ \pm 1}$. So the relation (1) holds. Let $g \in G_{i} \backslash H$ and $g^{\prime} \in G_{j} \backslash H$ with $i, j \in I_{0}$. Then

$$
T_{g}^{*} T_{g^{\prime}}=p_{i} g^{-1} g^{\prime} p_{j}=g^{-1} \chi_{g\left(\Omega \backslash Y_{i}\right)} \chi_{g^{\prime}\left(\Omega \backslash Y_{j}\right)} g^{\prime}=\delta_{i, j} \delta_{g H, g^{\prime} H} p_{i} g^{-1} g^{\prime} .
$$

Moreover it follows from $\Omega \backslash Y_{i}=\bigcup_{j \neq i} Y_{j}$ that

$$
\begin{aligned}
T_{g}^{*} T_{g}=\chi_{\Omega \backslash Y_{i}} & =\sum_{j \neq i} \chi_{Y_{j}} \\
& =\sum_{j \in I_{0}, j \neq i} \sum_{g \in \Omega_{j} \backslash\{e\}} \chi_{g\left(\Omega \backslash Y_{j}\right)}+\sum_{j \in I \backslash I_{0}} \chi_{a_{j}\left(\Omega \backslash Y_{j}\right)}+\chi_{a_{j}^{-1}\left(\Omega \backslash Y_{j}\right)} \sum_{j \in I_{0}, j \neq i} \sum_{g \in \Omega_{j} \backslash\{e\}} g^{-1}+\sum_{j \in I \backslash I_{0}} p_{j}^{+}+p_{j}^{-} \\
& =\sum_{j \in I_{0}, j \neq i} \sum_{g \in \Omega_{j} \backslash\{e\}} T_{g} T_{g}^{*}+\sum_{j \in I \backslash I_{0}} T_{a_{j}} T_{a_{j}}^{*}+T_{a_{j}^{-1}} T_{a_{j}^{-1}}^{*} .
\end{aligned}
$$

For all other cases, we can also check the relations (2) and (3) by similar calculations. Since $\Omega$ is the disjoint union of $Y_{i}$, we have (4). Note that $g, p_{i}, p_{i}^{ \pm} \in$ $C^{*}\left(T_{g}, V_{h}\right)$. Moreover, since the family $\left\{\gamma\left(\Omega \backslash Y_{i}\right) \mid \gamma \in \Gamma, i \in I\right\} \cup\left\{\gamma Y_{i}^{ \pm} \mid \gamma \in\right.$ $\left.\Gamma, i \in I \backslash I_{0}\right\}$ generates the topology of $\Omega$, we have $C(\Omega) \rtimes \Gamma=C^{*}\left(T_{g}, V_{h}\right)$. By the universality of $\mathcal{O}_{\Gamma}$, there exists a canonical surjective $*$-homomorphism of $\mathcal{O}_{\Gamma}$ onto $C(\Omega) \rtimes \Gamma$, sending $S_{g}$ to $T_{g}$ and $U_{h}$ to $V_{h}$.

Conversely, let $q_{i}=\sum_{j \neq i} P_{j}$ and $q_{i}^{ \pm}=S_{a_{i}^{ \pm 1}} S_{a_{i}^{ \pm 1}}^{*}$. Let

$$
\begin{cases}w_{g}=S_{g}+\sum_{g^{\prime} \in \Omega_{i} \backslash H \cup g^{-1} H} S_{g g^{\prime}} S_{g^{\prime}}^{*}+S_{g}^{*} & \text { for } g \in G_{i} \backslash H, i \in I_{0}, \\ w_{a_{i}}=S_{a_{i}}+S_{a_{i}^{-1}}^{*} & \text { for } i \in I \backslash I_{0}, \\ w_{h}=U_{h} & \text { for } h \in H .\end{cases}
$$


We will check that $w_{g}$ are unitaries for $g \in G_{i} \backslash H$ with $i \in I_{0}$. If $g^{\prime} \in$ $\Omega_{i} \backslash H \cup g^{-1} H$, then $g g^{\prime} H=\gamma H$ for some $\gamma \in \Omega_{i} \backslash\{e, g\}$. Hence

$$
\begin{aligned}
& w_{g} w_{g}^{*} \\
& =\left(S_{g}+\sum_{g^{\prime} \in \Omega_{i} \backslash H \cup g^{-1} H} S_{g g^{\prime}} S_{g^{\prime}}^{*}+S_{g^{-1}}^{*}\right)\left(S_{g}+\sum_{g^{\prime} \in \Omega_{i} \backslash H \cup g^{-1} H} S_{g g^{\prime}} S_{g^{\prime}}^{*}+S_{g^{-1}}^{*}\right)^{*} \\
& =S_{g} S_{g}^{*}+\sum_{g^{\prime} \in \Omega_{i} \backslash H \cup g^{-1} H} S_{g^{\prime}}^{*} S_{g^{\prime}} S_{g g^{\prime}}^{*}+S_{g^{-1}}^{*} S_{g^{-1}} \\
& =P_{g}+\sum_{g^{\prime} \in \Omega_{i} \backslash\{e, g\}} P_{g^{\prime}}+Q_{g}=1 .
\end{aligned}
$$

Similarly, we have $w_{g}^{*} w_{g}=1$. For the other case, we can check in the same way.

If $i \in I_{0}, \tau \in \Omega_{i} \backslash\{e\}$ then

$$
\begin{aligned}
\sum_{g \in \Omega_{i}} w_{g} q_{i} w_{g}^{*} & =\sum_{g \in \Omega_{i}}\left(S_{g}+\sum_{g^{\prime} \in \Omega_{i} \backslash H \cup g^{-1} H} S_{g g^{\prime}} S_{g^{\prime}}^{*}+S_{g^{-1}}^{*}\right) S_{\tau}^{*} S_{\tau} w_{g}^{*} \\
& =\sum_{g \in \Omega_{i}} S_{g} S_{\tau}^{*} S_{\tau}\left(S_{g}^{*}+\sum_{g^{\prime} \in \Omega_{i} \backslash H \cup g^{-1} H} S_{g} S_{g g^{\prime}}^{*}+S_{g^{-1}}\right) \\
& =\sum_{g \in \Omega_{i}} S_{g} S_{\tau}^{*} S_{\tau} S_{g}^{*}=1 .
\end{aligned}
$$

For $i \in I \backslash I_{0}$, we have $q_{i}^{+}+w_{a_{i}} q_{i}^{-} w_{a_{i}}^{*}=1$ and $q_{i}^{+}+q_{i}^{-}+q_{i}=1$ as well. Therefore the conjugates of the family $\left\{q_{i}, q_{i}^{ \pm}\right\}$by the elements of $\Gamma$ generate a commutative $C^{*}$-algebra. This is the image of a representation of $C(\Omega)$. Therefore $\left(q_{i}, w\right)$ gives a covariant representation of the $C^{*}$-dynamical system $(C(\Omega), \Gamma)$. Note that $\left(q_{i}, w_{g}\right)$ generates $\mathcal{O}_{\Gamma}$. Hence by the universality of the full crossed product $C(\Omega) \rtimes \Gamma$, there exists a canonical surjective $*$-homomorphism of $C(\Omega) \rtimes \Gamma$ onto $\mathcal{O}_{\Gamma}$. It is easy to show that the above two $*$-homomorphisms are the inverses of each other.

$$
\begin{array}{rlrl}
S_{g} & \mapsto g p_{i} & \mapsto & w_{g} Q_{g}=S_{g}, \\
S_{a_{i}^{ \pm 1}} & \mapsto a_{i}^{ \pm 1}\left(p_{i}+p_{i}^{ \pm}\right) & \mapsto w_{a_{i}^{ \pm 1}}\left(Q_{a_{i}^{ \pm 1}}+P_{a_{i}^{ \pm 1}}\right)=S_{a_{i}^{ \pm 1}}, \\
U_{h} & \mapsto \quad h \quad & \mapsto & U_{h} .
\end{array}
$$

We have shown the identification $\mathcal{O}_{\Gamma} \simeq C(\Omega) \rtimes \Gamma$. Since there exists a canonical surjective map of $C(\Omega) \rtimes \Gamma$ onto $C(\Omega) \rtimes_{r} \Gamma$, we have a surjective *-homomorphism of $\mathcal{O}_{\Gamma}$ onto $C(\Omega) \rtimes_{r} \Gamma$. Let $C(\Omega) \rtimes_{r} \Gamma=C^{*}\left(\tilde{\pi}\left(p_{i}\right), \lambda\right)$ where 
$\tilde{\pi}$ is the induced representation on the Hilbert space $l^{2}(\Gamma, \mathcal{H})$ by the universal representation $\pi$ of $C(\Omega)$ on a Hilbert space $\mathcal{H}$ and $\lambda$ is the unitary representation of $\Gamma$ on $l^{2}(\Gamma, \mathcal{H})$ such that $\left(\lambda_{s} x\right)(t)=x\left(s^{-1} t\right)$ for $x \in l^{2}(\Gamma, \mathcal{H})$. By the uniqueness theorem for $\mathcal{O}_{\Gamma}$, it suffices to check

$$
C^{*}\left(\tilde{\pi}\left(\chi_{Y_{i}}\right) \lambda_{h} \tilde{\pi}\left(\chi_{Y_{i}}\right)\right) \simeq C^{*}(H)
$$

But the unitary representation $\tilde{\pi}\left(\chi_{Y_{i}}\right) \lambda_{h} \tilde{\pi}\left(\chi_{Y_{i}}\right)$ is quasi-equivalent to the left regular representation of $H$. This completes the proof of the theorem.

In [27], Serre defined the tree $G_{T}$, on which $\Gamma$ acts. In an appendix, we will give the definition of the tree $G_{T}=(V, E)$ where $V$ is the set of vertices and $E$ is the set of edges. We denote the corresponding natural boundary by $\partial G_{T}$. We also show how to construct boundaries of trees in the appendix. (See Furstenberg [17] and Freudenthal [16] for details.)

Proposition 5.4. $\quad$ The space $\partial G_{T}$ is homeomorphic to $\Omega$ and the above two actions of $\Gamma$ on $\partial G_{T}$ and $\Omega$ are conjugate.

Proof. We define a map $\psi$ from $\partial G_{T}$ to $\Omega$. First we assume that $I=$ $\{1,2\}$. The corresponding tree $G_{T}$ consists of the vertex set $V=\Gamma / G_{1} \amalg \Gamma / G_{2}$ and the edge set $E=\Gamma / H$. For $\omega \in \partial G_{T}$, we can identify $\omega$ with an infinite chain $\left\{G_{i_{1}}, g_{1} G_{i_{2}}, g_{1} g_{2} G_{i_{3}}, \ldots\right\}$ with $g_{k} \in \Omega_{i_{k}} \backslash\{e\}$ and $i_{1} \neq i_{2} \neq \cdots$. Then we define $\psi(\omega)=\left[x(n)=g_{i_{n}}\right]$. We will recall the definition of the corresponding tree $G_{T}$, in general, on the appendix, (see [27]). Similarly, we can identify $\omega \in \partial G_{T}$ with an infinite chain $\left\{G_{0}, G_{i_{1}}, g_{1} G_{0}, g_{1} G_{i_{2}}, g_{1} g_{2} G_{0}, \ldots\right\}$. Moreover we may ignore vertices $\gamma G_{0}$ for an infinite chain $\omega$,

$$
\left\{G_{0}, G_{i_{1}},\left(g_{1} G_{0} \rightarrow \text { ignoring }\right), g_{1} G_{i_{2}},\left(g_{1} g_{2} G_{0} \rightarrow \text { ignoring }\right), g_{1} g_{2} G_{i_{3}}, \ldots\right\} .
$$

Therefore, we define a map $\psi$ of $\partial G_{T}$ to $\Omega$ by

$$
\psi(\omega)=\left[x(n)=g_{n}\right] .
$$

The pull-back by $\psi$ of any open set of $\partial G_{T}$ is an open set on $\Omega$. It follows that $\psi$ is a homeomorphism. The two actions on $\partial G_{T}$ and $\Omega$ are defined by left multiplication. So it immediately follows that these actions are conjugate.

It is known that $\Gamma$ is a hyperbolic group (see a proof in the appendix, where we recall the notion of hyperbolicity for finitely generated groups as introduced by Gromov e.g. see [18]). Let $S=\left\{\bigcup_{i \in I} G_{i}\right\}$ and $G(\Gamma, S)$ be the Cayley graph of $\Gamma$ with the word metric $d$. Let $\partial \Gamma$ be the hyperbolic boundary. 
Proposition 5.5. The hyperbolic boundary $\partial \Gamma$ is homeomorphic to $\Omega$ and the actions of $\Gamma$ are conjugate.

Proof. We can define a map $\psi$ from $\Omega$ to $\partial \Gamma$ by $(x(n)) \mapsto\left[x_{n}=x(1) \cdots\right.$ $x(n)$ ]. Indeed, since $\left\langle x_{n} \mid x_{m}\right\rangle=\min \{n, m\} \rightarrow \infty(n, m \rightarrow \infty)$, it is well-defined. For $x \neq y$ in $\Omega$, there exists $k$ such that $x(k) \neq y(k)$. Then $\langle\psi(x) \mid \psi(y)\rangle \leq k+1$, which shows injectivity. Let $\left(x_{n}\right) \in \partial \Gamma$. Suppose that $x_{n}=g_{n(1)} \cdots g_{n\left(k_{n}\right)} h_{n}$ for some $g_{l} \in \bigcup_{i} \Omega_{i} \backslash\{e\}$ with $n(1) \neq \cdots \neq n\left(k_{n}\right)$. If $g_{n(1)}=g_{m(1)}, \ldots, g_{n(l)}=$ $g_{m(l)}$ and $g_{n(l+1)} \neq g_{m(l+1)}$, then we set $a_{n, m}=g_{n(1)} \cdots g_{n(l)}=g_{m(1)} \cdots g_{m(l)}$. So we have

$$
\left\langle x_{n} \mid x_{m}\right\rangle \leq d\left(e, a_{n, m}\right)+1 \rightarrow \infty(n, m \rightarrow \infty) .
$$

Therefore we can choose sequences $n_{1}<n_{2}<\cdots$, and $m_{1}<m_{2}<\cdots$, such that $a_{n_{k}, m_{k}}$ is a sub-word of $a_{n_{k+1}, m_{k+1}}$. Then a sequence $\left\{g_{n_{k}(1)}, \ldots, g_{n_{k}(l)}\right.$, $\left.g_{n_{k+1}(l+1)}, \ldots\right\}$ is mapped to $\left(x_{n}\right)$ by $\psi$. We have proved that $\psi$ is surjective. The pull-back of any open set in $\partial \Gamma$ is an open set in $\Omega$. So $\psi$ is continuous. Since $\Omega, \partial \Gamma$ are compact Hausdorff spaces, $\psi$ is a homeomorphism. Again, the two actions on $\Omega$ and $\partial \Gamma$ are defined by left multiplication and hence are conjugate.

Remark. Since the action of $\Gamma$ on $\partial \Gamma$ depends only on the group structure of $\Gamma$ in [18], the above proposition shows that $\mathcal{O}_{\Gamma}$ is, up to isomorophism, independent of the choice of generators of $\Gamma$.

\section{$\S 6$. Nuclearity, Simplicity and Pure Infiniteness of $\mathcal{O}_{\Gamma}$}

We first begin by reviewing the crossed product $B \rtimes \mathbb{N}$ of a $C^{*}$-algebra $B$ by a $*$-endomorphism; this construction was first introduced by Cuntz [6] to describe the Cuntz algebra $\mathcal{O}_{n}$ as the crossed product of UHF algebras by *-endomorphisms. See Stacey's paper [29] for a more detailed discussion. Suppose that $\rho$ is an injective $*$-endomorphism on a unital $C^{*}$-algebra $B$. Let $\bar{B}$ be the inductive limit $\underset{\lim }{\longrightarrow}(B \stackrel{\rho}{\longrightarrow} B)$ with the corresponding injective homo-

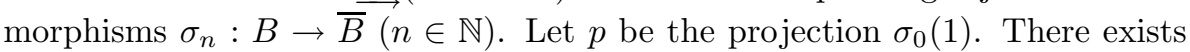
an automorphism $\bar{\rho}$ given by $\bar{\rho} \circ \sigma_{n}=\sigma_{n} \circ \rho$ with inverse $\sigma_{n}(b) \mapsto \sigma_{n+1}(b)$. Then the crossed product $B \rtimes_{\rho} \mathbb{N}$ is defined to be the hereditary $C^{*}$-algebra $p\left(\bar{B} \rtimes_{\bar{\rho}} \mathbb{Z}\right) p$. The map $\sigma_{0}$ induces an embedding of $B$ into $\bar{B}$. Therefore the canonical embedding of $\bar{B}$ into $\bar{B} \rtimes_{\bar{\rho}} \mathbb{Z}$ gives an embedding $\pi: B \rightarrow B \rtimes_{\rho} \mathbb{N}$. Moreover the compression by $p$ of the implementing unitary is an isometry $V$ belonging to $B \rtimes_{\rho} \mathbb{N}$ satisfying

$$
V \pi(b) V^{*}=\pi(\rho(b)) .
$$


In fact, $B \rtimes_{\rho} \mathbb{N}$ is also the universal $C^{*}$-algebra generated by a copy $\pi(B)$ of $B$ and an isometry $V$ satisfying the above relation. If $B$ is nuclear, then so is $B \rtimes_{\rho} \mathbb{N}$.

\section{Proposition 6.1.}

$$
\mathcal{O}_{\Gamma} \simeq \mathcal{O}_{\Gamma}^{\mathbb{T}} \rtimes_{\rho} \mathbb{N}
$$

In particular, $\mathcal{O}_{\Gamma}$ is nuclear.

Proof. We fix $g_{i} \in G_{i} \backslash H$ for all $i \in I$. We can choose projections $e_{i}$ which are sums of projections $P_{g}$ such that $e_{i} \leq Q_{g_{i}}$ and $\sum_{i \in I} e_{i}=1$. Then $V=\sum_{i \in I} S_{g_{i}} e_{i}$ is an isometry in $\mathcal{O}_{\Gamma}$.

We claim that $V \mathcal{O}_{\Gamma}^{\mathbb{T}} V^{*} \subseteq \mathcal{O}_{\Gamma}^{\mathbb{T}}$ and $\mathcal{O}_{\Gamma}=C^{*}\left(\mathcal{O}_{\Gamma}^{\mathbb{T}}, V\right)$. Let $a \in \mathcal{O}_{\Gamma}^{\mathbb{T}}$. It is obvious that $V a V^{*} \in \mathcal{O}_{\Gamma}^{\mathbb{T}}$ and $C^{*}\left(\mathcal{O}_{\Gamma}^{\mathbb{T}}, V\right) \subseteq \mathcal{O}_{\Gamma}$. To show the second claim, it suffices to check that $S_{\mu} P_{i} S_{\nu}^{*} \in \mathcal{O}_{\Gamma}$ for all $\mu, \nu$ and $i$. If $|\mu|=|\nu|$, we have $S_{\mu} P_{i} S_{\nu}^{*} \in \mathcal{O}_{\Gamma}^{\mathbb{T}}$. If $|\mu| \neq|\nu|$, then we may assume $|\mu|<|\nu|$. Let $|\nu|-|\mu|=k$. Thus $S_{\mu} P_{i} S_{\nu}^{*}=\left(V^{*}\right)^{k} V^{k} S_{\mu} P_{i} S_{\nu}^{*}$ and $V^{k} S_{\mu} P_{i} S_{\nu}^{*} \in \mathcal{O}_{\Gamma}^{\mathbb{T}}$. This proves our claim.

We define a $*$-endomorphism $\rho$ of $\mathcal{O}_{\Gamma}^{\mathbb{T}}$ by $\rho(a)=V a V^{*}$ for $a \in \mathcal{O}_{\Gamma}^{\mathbb{T}}$. Thanks to the universality of the crossed product $\mathcal{O}_{\Gamma}^{\mathbb{T}} \rtimes_{\rho} \mathbb{N}$, we obtain a canonical surjective $*$-homomorphism $\sigma$ of $\mathcal{O}_{\Gamma}^{\mathbb{T}} \rtimes_{\rho} \mathbb{N}$ onto $C^{*}\left(\mathcal{O}_{\Gamma}^{\mathbb{T}}, V\right)$. Since $\mathcal{O}_{\Gamma}^{\mathbb{T}} \rtimes_{\rho} \mathbb{N}$ has the universal property, there also exists a gauge action $\beta$ on $\mathcal{O}_{\Gamma}^{\mathbb{T}} \rtimes_{\rho} \mathbb{N}$. Let $\Psi$ be the corresponding canonical conditional expectation of $\mathcal{O}_{\Gamma}^{\mathbb{T}} \rtimes_{\rho} \mathbb{N}$ onto $\mathcal{O}_{\Gamma}^{\mathbb{T}}$. Suppose that $a \in \operatorname{ker} \sigma$. Then $\sigma\left(a^{*} a\right)=0$. Since $\alpha \circ \sigma=\sigma \circ \beta$, we have $\sigma \circ \Psi\left(a^{*} a\right)=0$. The injectivity of $\sigma$ on $\mathcal{O}_{\Gamma}^{\mathbb{T}}$ implies $\Psi\left(a^{*} a\right)=0$ and hence $a^{*} a=0$ and $a=0$. It follows that $\mathcal{O}_{\Gamma} \simeq \mathcal{O}_{\Gamma}^{\mathbb{T}} \rtimes_{\rho} \mathbb{N}$.

In Section 2, we reviewed the notion of amenability for discrete group actions. The following is a special case of [1]

Corollary 6.2. The action of $\Gamma$ on $\partial \Gamma$ is amenable.

Proof. This follows from Theorem 2.2 and the above proposition.

We also have a partial result of [20], [9], [10] and [11].

Corollary 6.3. $\quad$ The reduced group $C^{*}$-algebra $C_{r}^{*}(\Gamma)$ is exact.

Proof. It is well-known that every $C^{*}$-subalgebra of an exact $C^{*}$-algebra is exact; see Wassermann's monograph [31]. Therefore the inclusion $C_{r}^{*}(\Gamma) \subset \mathcal{O}_{\Gamma}$ implies exactness.

Finally we give a sufficient condition for the simplicity and pure infiniteness of $\mathcal{O}_{\Gamma}$. 
Corollary 6.4. Suppose that $\Gamma={ }^{*}{ }_{H} G_{i}$ satisfies the following condition:

There exists at least one element $j \in I$ such that

$$
\bigcap_{i \neq j} N_{i}=\{e\},
$$

where $N_{i}=\bigcap_{g \in G_{i}} g H g^{-1}$.

Then $\mathcal{O}_{\Gamma}$ is simple and purely infinite.

Proof. We first claim that for any $\mu \in \Delta$ and $|g|=1$ with $|\mu g|=|\mu|+1$,

$$
\mu H \mu^{-1} \cap H \supseteq \mu g H g^{-1} \mu^{-1} \cap H
$$

Suppose that $\mu=\mu_{1} \cdots \mu_{n}$ such that $\mu_{k} \in \Omega_{i_{k}}$ with $\mu_{1} \neq \cdots \neq \mu_{n}$ and $g \in G_{i}$ with $i \neq i_{n}$. We first assume that $\mu=\mu_{1}$. If $\mu g h g^{-1} \mu^{-1} \in \mu g \mathrm{Hg}^{-1} \mu^{-1} \cap H$, then $g h g^{-1} \in \mu^{-1} H \mu \subseteq G_{i_{1}}$. Thus $g h g^{-1} \in G_{i} \cap G_{i_{1}}$ implies $g h g^{-1} \in H$. Next we assume that $|\mu|>1$. If $\mu g h g^{-1} \mu^{-1} \in \mu g H g^{-1} \mu^{-1} \cap H$, then

$$
\mu_{2} \cdots \mu_{n} g h g^{-1} \mu_{k}^{-1} \cdots \mu_{2}^{-1} \in \mu_{1}^{-1} H \mu_{1} \subseteq G_{i_{1}} .
$$

Thus $\left|\mu_{2} \cdots \mu_{n} g h g^{-1} \mu_{k}^{-1} \cdots \mu_{2}^{-1}\right| \leq 1$ implies $g h g^{-1} \in H$. This proves the claim.

Let $\left\{S_{g}, U_{h}\right\}$ be any family satisfying the relations (1), (2), (3) and (4). By the uniqueness theorem, it is enough to show that $C^{*}\left(P_{i} U_{h} P_{i} \mid h \in H\right) \simeq$ $C^{*}(H)$ for any $i \in I$. We next claim that there exists $\nu \in \Gamma$ such that the initial letter of $\nu$ belongs to $\Omega_{i}$ and $\left\{U_{h} S_{\nu}\right\}_{h \in H}$ have mutually orthogonal ranges.

Let $g \in \Omega_{i}$. If $g H^{-1} \cap H=\{e\}$, then it is enough to set $\nu=g$. Now suppose that there exists some $h \in g H^{-1} \cap H$ with $h \neq e$. We first assume that $i=j$. By the hypothesis, there exists some $i_{1} \in I$ such that $g^{-1} h g \notin N_{i_{1}}$ and $i \neq i_{1}$. Hence there exists $g_{1} \in \Omega_{i_{1}}$ such that $g^{-1} h g \notin g_{1} H g_{1}^{-1}$ and so $h \notin g g_{1} H g_{1}^{-1} g^{-1}$. If $g g_{1} H g_{1}^{-1} g^{-1} \cap H=\{e\}$, then it is enough to put $\nu=g g_{1}$. If not, we set $\gamma_{1}=g_{1} g_{1}^{\prime}$ for some $g_{1}^{\prime} \in \Omega_{j}$. By the first part of the proof, we have

$$
g H g^{-1} \cap H \supsetneqq \mu \gamma_{1} H \gamma_{1}^{-1} \mu^{-1} \cap H .
$$

Since $H$ is finite, we can inductively obtain $\gamma_{1}, \gamma_{2}, \ldots \gamma_{n}$ satisfying $g H g^{-1} \cap H \supsetneqq g \gamma_{1} H \gamma_{1}^{-1} g^{-1} \cap H \supsetneqq \cdots \supsetneqq g \gamma_{1} \cdots \gamma_{n} H \gamma_{n}^{-1} \cdots \gamma_{1}^{-1} g^{-1} \cap H=\{e\}$.

Then we set $\nu=g \gamma_{1} \cdots \gamma_{n}$. If $i \neq j$, we can carry out the same arguments by replacing $g$ by $\gamma=g g_{j}$ for some $g_{j} \in \Omega_{j}$. Hence from the identification 
$U_{h} S_{\nu} \leftrightarrow \delta_{h} \in l^{2}(H)$, it follows that the unitary representation $P_{i} U_{h} P_{i}$ is quasiequivalent to the left regular representation of $H$. Thus $\mathcal{O}_{\Gamma}$ is simple.

In Section 5, we have proved that $\mathcal{O}_{\Gamma} \simeq C(\Omega) \rtimes_{r} \Gamma$. We show that the action of $\Gamma$ on $\Omega$ is the strong boundary action (see Preliminaries). Let $U, V$ be any non-empty open sets in $\Omega$. There exists some open set $O=\{(x(n)) \in$ $\left.\Omega \mid x(1)=g_{1}, \ldots, x(k)=g_{k}\right\}$ which is contained in $V$. We may also assume that $U^{c}$ is an open of the form $\left\{(x(n)) \in \Omega \mid x(1)=\gamma_{1}, \ldots, x(m)=\gamma_{m}\right\}$. Let $\gamma=g_{1} \cdots g_{k} \gamma_{m}^{-1} \cdots \gamma_{1}^{-1}$. Then we have $\gamma U^{c} \subset O \subset V$. Since $C(\Omega) \rtimes_{r} \Gamma$ is simple, it follows from [3] that the action of $\Gamma$ is topological free. Therefore it follows from Theorem 2.4 that $C(\Omega) \rtimes_{r} \Gamma$, namely $\mathcal{O}_{\Gamma}$, is purely infinite.

Remark. We gave a sufficient condition for $\mathcal{O}_{\Gamma}$ to be simple. However, we can completely determine the ideal structure of $\mathcal{O}_{\Gamma}$ with further effort. Indeed, we will obtain a matrix $A_{\Gamma}$ to compute K-groups of $\mathcal{O}_{\Gamma}$ in the next section. The same argument as in [7] also works for the ideal structure of $\mathcal{O}_{\Gamma}$. For Cuntz-Krieger algebras, we need to assume that corresponding matrices have the condition (II) of [7] to apply the uniqueness theorem. Since we have another uniqueness theorem for our algebras, we can always apply the ideal structure theorem.

Let $\Sigma=I \times\{1, \ldots, r\}$ be a finite set, where $r$ is the number of all irreducible unitary representations of $H$. For $x, y \in \Sigma$, we define $x \geq y$ if there exists a sequence $x_{1}, \ldots, x_{m}$ of elements in $\Sigma$ such that $x_{1}=x, x_{m}=y$ and $A_{\Gamma}\left(x_{a}, x_{a+1}\right) \neq 0(a=1, \ldots, m-1)$. We call $x$ and $y$ equivalent if $x \geq y \geq x$ and write $\Gamma_{A_{\Gamma}}$ for the partially ordered set of equivalence classes of elements $x$ in $\Sigma$ for which $x \geq x$. A subset $K$ of $\Gamma_{A_{\Gamma}}$ is called hereditary if $\gamma_{1} \geq \gamma_{2}$ and $\gamma_{1} \in K$ implies $\gamma_{2} \in K$. Let

$$
\Sigma(K)=\left\{x \in \Sigma \mid x_{1} \geq x \geq x_{2} \quad \text { for some } \quad x_{1}, x_{2} \in \bigcup_{\gamma \in K} \gamma\right\} .
$$

We denote by $I_{K}$ the closed ideal of $\mathcal{O}_{\Gamma}$ generated by projections $P(i, k)$, which is defined in the next section, for all $(i, k) \in \Sigma(K)$.

Theorem 6.5 ([7, Theorem 2.5]). The map $K \mapsto I_{K}$ is an inclusion preserving bijection of the set of hereditary subsets of $\Gamma_{A_{\Gamma}}$ onto the set of closed ideals of $\mathcal{O}_{\Gamma}$.

\section{$\S 7 . \quad K$-theory for $\mathcal{O}_{\Gamma}$}

In this section we give explicit formulae of the $K$-groups of $\mathcal{O}_{\Gamma}$. We have described $\mathcal{O}_{\Gamma}$ as the crossed product $\mathcal{O}_{\Gamma}^{\mathbb{T}} \rtimes \mathbb{N}$ in Section 6 . So to apply the 
Pimsner-Voiculescu exact sequence [24], we need to compute the $K$-groups of the $A F$-algebra $\mathcal{O}_{\Gamma}^{\mathbb{T}}$. We assume that each $G_{i}$ is finite for simplicity throughout this section. We can also compute the $K$-groups for general cases by essentially the same arguments. Recall that the fixed-point algebra is described as follows:

$$
\begin{aligned}
& \mathcal{O}_{\Gamma}^{\mathbb{T}}=\overline{\bigcup_{n \geqq 0} \mathcal{F}_{n}}, \\
& \mathcal{F}_{n}=\oplus_{i \in I} \mathcal{F}_{n}^{i} .
\end{aligned}
$$

For each $n$, we consider a direct summand of $\mathcal{F}_{n}$, which is

$$
\mathcal{F}_{n}^{i}=C^{*}\left(S_{\mu} P_{i} U_{h} P_{i} S_{\nu}^{*}|h \in H,| \mu|=| \nu \mid=n\right),
$$

and the embedding $\mathcal{F}_{n}^{i} \hookrightarrow \mathcal{F}_{n+1}$ is given by

$$
\begin{aligned}
S_{\mu} P_{i} U_{h} P_{i} S_{\nu}^{*} & =\sum_{g \in \Omega_{i} \backslash\{e\}} S_{\mu} U_{h}\left(S_{g} Q_{g} S_{g}^{*}\right) S_{\nu}^{*} \\
& =\sum_{g} \sum_{i^{\prime} \neq i} S_{\mu} S_{h g} P_{i^{\prime}} S_{\nu g}^{*} .
\end{aligned}
$$

Let $\left\{\chi_{1}, \ldots, \chi_{r}\right\}$ be the set of characters corresponding with all irreducible unitary representations of the finite group $H$ with degrees $n_{1}, \ldots, n_{r}$. Then we have the identification $C^{*}(H) \simeq M_{n_{1}}(\mathbb{C}) \oplus \cdots \oplus M_{n_{r}}(\mathbb{C})$. We can write a unit $p_{k}$ of the $k$-th component $M_{n_{k}}(\mathbb{C})$ of $C^{*}(H)$ as follows:

$$
p_{k}=\frac{n_{k}}{|H|} \sum_{h \in H} \overline{\chi_{k}(h)} U_{h}
$$

Suppose that for $i \neq j$,

$$
\begin{gathered}
\mathcal{F}_{n}^{i} \simeq M_{N(n, i)}(\mathbb{C}) \otimes C^{*}(H), \\
\mathcal{F}_{n+1}^{j} \simeq M_{N(n+1, j)}(\mathbb{C}) \otimes C^{*}(H) .
\end{gathered}
$$

Now we compute each embedding of $\mathcal{F}_{n}^{i} \hookrightarrow \mathcal{F}_{n+1}^{j}$,

$$
M_{N(n, i)}(\mathbb{C}) \otimes M_{n_{i}}(\mathbb{C}) \hookrightarrow M_{N(n+1, j)}(\mathbb{C}) \otimes M_{n_{j}}(\mathbb{C})
$$

at the $K$-theory level. $P(i, k)$ denotes $P_{i} p_{k} P_{i}$. Let $P$ be the projection $e \otimes 1$ in $M_{N(n, i)}(\mathbb{C}) \otimes M_{n_{k}}(\mathbb{C})$ given by

$$
P=S_{\mu} P(i, k) S_{\mu}^{*} \quad \text { for some } \mu \in \Delta_{n},
$$


where $e$ is a minimal projection in the matrix algebras, and $Q$ be the unit of $M_{N(n+1, j)}(\mathbb{C}) \otimes M_{n_{l}}(\mathbb{C})$ given by

$$
Q=\sum_{\nu \in \Delta_{n+1}} S_{\nu} P(j, l) S_{\nu}^{*}
$$

At the $K$-theory level, we have $[P]=n_{k}[e]$. Hence it suffices to compute $\operatorname{tr}(P Q) / n_{k}$, where tr is the canonical trace in the matrix algebras.

$$
\begin{aligned}
& \frac{\operatorname{tr}(P Q)}{n_{k}} \\
& =\operatorname{tr}\left(\frac{1}{n_{k}}\left(S_{\mu} P(i, k) S_{\mu}^{*}\right)\left(\sum_{\nu \in \Delta_{n+1}} S_{\nu} P(j, l) S_{\nu}^{*}\right)\right) \\
& =\operatorname{tr}\left(\frac{1}{|H|}\left(\sum_{h \in H} \overline{\chi_{k}(h)} S_{\mu} U_{h} P_{i} S_{\mu}^{*}\right)\left(\sum_{\nu \in \Delta_{n+1}} S_{\nu} P(j, l) S_{\nu}^{*}\right)\right) \\
& =\frac{1}{|H|} \operatorname{tr}\left(\sum_{h \in H} \frac{1}{\chi_{k}(h)}\left(\sum_{g \in \Omega_{i} \backslash\{e\}} \sum_{i^{\prime} \neq i} S_{\mu} S_{h g} P_{i^{\prime}} S_{\mu g}^{*}\right)\left(\sum_{\nu \in \Delta_{n+1}} S_{\nu} P(j, l) S_{\nu}^{*}\right)\right) \\
& =\frac{1}{|H|} \operatorname{tr}\left(\sum_{h \in H} \frac{1}{\chi_{k}(h)}\left(\sum_{g \in \Omega_{i} \backslash\{e\}} S_{\mu} S_{h g} P(j, l) S_{\mu g}^{*}\right)\right) \\
& =\frac{1}{|H|} \sum_{g \in \Omega_{i} \backslash\{e\}} \sum_{h \in H(g)} \frac{1}{\chi_{k}(h)} \operatorname{tr}\left(S_{\mu g} U_{g^{-1} h g} P(j, l) S_{\mu g}^{*}\right) \\
& =\frac{1}{|H|} \sum_{g \in \Omega_{i} \backslash\{e\}} \sum_{h \in H(g)} \frac{\chi_{k}(h)}{\chi_{l}}\left(g^{-1} h g\right),
\end{aligned}
$$

where $H(g)$ is the stabilizer of $g H$ by the left multiplication of $H$.

Now fix $x \in X_{i} \backslash\{e\}$. Let $\left\{g \in \Omega_{i} \mid H g H=H x H\right\}=\left\{g_{0}=x, g_{1}, \ldots\right.$, $\left.g_{m-1}\right\}$. Then there exists $h_{1}, h_{1}^{\prime}, \ldots, h_{m-1}, h_{m-1}^{\prime} \in H$ such that $h_{1} x=$ $g_{1} h_{1}^{\prime}, \ldots, h_{m-1} x=g_{m-1} h_{m-1}^{\prime}$. Note that $h_{s} H(x) h_{s}^{-1}=H\left(g_{s}\right)$ for $s=1$, $\ldots, m-1$. Since $\chi_{k}, \chi_{l}$ are class functions, we have

$$
\begin{aligned}
\frac{\operatorname{tr}(P Q)}{n_{k}} & =\frac{1}{|H|} \sum_{x \in X_{i}}\left(\sum_{s=1}^{m-1} \sum_{h \in H(x)} \overline{\chi_{k}\left(h_{s} h h_{s}^{-1}\right)} \chi_{l}\left(h_{s}^{\prime} x^{-1} h_{s}^{-1} \cdot h_{s} h h_{s}^{-1} \cdot h_{s} x h_{s}^{\prime-1}\right)\right) \\
& =\frac{1}{|H|} \sum_{x \in X_{i}}\left(\sum_{s=1}^{m-1} \sum_{h \in H(x)} \overline{\chi_{k}\left(h_{s} h h_{s}^{-1}\right)} \chi_{l}\left(h_{s}^{\prime} x^{-1} h x h_{s}^{\prime-1}\right)\right)
\end{aligned}
$$




$$
\begin{aligned}
& =\frac{1}{|H|} \sum_{x \in X_{i}}\left(\sum_{s=1}^{m-1} \sum_{h \in H(x)} \overline{\chi_{k}(h)} \chi_{l}\left(x^{-1} h x\right)\right) \\
& =\frac{1}{|H|} \sum_{x \in X_{i}}\left(\sum_{s=1}^{m-1} \sum_{h \in H(x)} \overline{\chi_{k}(h)} \chi_{l}^{x}(h)\right) \\
& =\sum_{x \in X_{i}}\left(\frac{|H(x)|}{|H|} \sum_{s=1}^{m-1}\left\langle\chi_{k}, \chi_{l}^{x}\right\rangle_{H(x)}\right) \\
& =\sum_{x \in X_{i}}\left\langle\chi_{k}, \chi_{l}^{x}\right\rangle_{H(x)}
\end{aligned}
$$

where

$$
\begin{aligned}
\chi_{l}^{x}(h) & =\chi_{l}\left(x^{-1} h x\right) \\
\left\langle\chi_{k}, \chi_{l}^{x}\right\rangle_{H(x)} & =\frac{1}{|H(x)|} \sum_{h \in H(x)} \overline{\chi_{k}(h)} \chi_{l}^{x}(h) .
\end{aligned}
$$

Let $A_{\Gamma}((j, l),(i, k))=\sum_{x \in X_{i} \backslash\{e\}}\left\langle\chi_{k}, \chi_{l}^{x}\right\rangle_{H(x)}$ for $i \neq j$ and $A_{\Gamma}((i, k),(i, l))$ $=0$ for $1 \leq k, l \leq r$. Then we describe the embedding $\mathcal{F}_{n}^{i} \hookrightarrow \mathcal{F}_{n+1}^{j}$ at the $K$ theory level by the matrix $\left[A_{\Gamma}((i, k),(j, l))\right]_{1 \leq k, l \leq r}$. Let $A_{\Gamma}=\left[A_{\Gamma}((i, k),(j, l))\right]$. We have the following lemma.

\section{Lemma 7.1.}

$$
\begin{gathered}
K_{0}\left(\mathcal{O}_{\Gamma}^{\mathbb{T}}\right)=\stackrel{\lim }{\longrightarrow}\left(\mathbb{Z}^{N} \stackrel{A_{\Gamma}}{\longrightarrow} \mathbb{Z}^{N}\right) \\
K_{1}\left(\mathcal{O}_{\Gamma}^{\mathbb{T}}\right)=0
\end{gathered}
$$

where $N=|I| r$.

We can compute the $K$-groups of $\mathcal{O}_{\Gamma}$ by using the Pimsner-Voiculescu sequence with essentially the same argument as in the Cuntz-Krieger algebra case (see [7]).

\section{Theorem 7.2.}

$$
\begin{aligned}
& K_{0}\left(\mathcal{O}_{\Gamma}\right)=\mathbb{Z}^{N} /\left(1-A_{\Gamma}\right) \mathbb{Z}^{N} \\
& K_{1}\left(\mathcal{O}_{\Gamma}\right)=\operatorname{Ker}\left\{1-A_{\Gamma}: \mathbb{Z}^{N} \rightarrow \mathbb{Z}^{N}\right\} \quad \text { on } \mathbb{Z}^{N}
\end{aligned}
$$

Proof. It suffices to compute the $K$-groups of $\overline{\mathcal{O}}_{\gamma}=\overline{\mathcal{O}}_{\Gamma}^{\mathbb{T}} \rtimes_{\bar{\rho}} \mathbb{Z}$. We represent the inductive limit

$$
\stackrel{\lim }{\longrightarrow}\left(\mathbb{Z}^{N} \stackrel{A_{\Gamma}}{\longrightarrow} \mathbb{Z}^{N}\right)
$$


as the set of equivalence classes of $x=\left(x_{1}, x_{2}, \ldots\right)$ such that $x_{k} \in \mathbb{Z}^{N}$ with $x_{k+1}=A\left(x_{k}\right)$. If $S$ is a partial isometry in $\mathcal{O}_{\Gamma}$ such that $\alpha_{z}(S)=z S$ and $P$ is a projection in $\mathcal{O}_{\Gamma}^{\mathbb{T}}$ with $P \leq S^{*} S$, then $[\rho(P)]=\left[V P V^{*}\right]=\left[\left(V S^{*} S\right) P\left(V S^{*} S\right)^{*}\right]$ $=\left[S P S^{*}\right]$ in $K_{0}\left(\mathcal{O}_{\Gamma}^{\mathbb{T}}\right)$. Recall that

$$
p_{k}=\frac{n_{k}}{|H|} \sum_{h \in H} \overline{\chi_{k}(h)} U_{h} .
$$

Let $P=S_{\mu} P(i, k) S_{\mu}^{*}$ for some $\mu \in \Delta_{n}$. If $\mu=\mu_{1} \cdots \mu_{n}$, then

$$
\begin{aligned}
{\left[\bar{\rho}^{-1}(P)\right] } & =\left[S_{\mu_{1}}^{*} P S_{\mu_{1}}\right] \\
& =\left[\frac{n_{k}}{|H|} \sum_{h \in H} \overline{\chi_{k}(h)}\left(S_{\mu_{2}} \cdots S_{\mu_{n}} P_{i} U_{h} P_{i} S_{\mu_{n}} \cdots S_{\mu_{2}}^{*}\right)\right] \\
& =\cdots \\
& =\sum_{j \neq i} \sum_{l=1}^{r} n_{i}\left(\sum_{x \in X_{i} \backslash\{e\}}\left\langle\chi_{k}, \chi_{l}^{x}\right\rangle\left[e_{l}\right]\right)
\end{aligned}
$$

where the $e_{l}$ are non-zero minimal projections for $1 \leq l \leq r$. Thus it follows that $\bar{\rho}_{*}^{-1}$ is the shift on $K_{0}\left(\overline{\mathcal{O}}_{\Gamma}^{\mathbb{T}}\right)$. We denote the shift by $\sigma$. If $x=\left(x_{1}, x_{2}, x_{3}, \ldots\right) \in$ $K_{0}\left(\overline{\mathcal{O}}_{\Gamma}^{\mathbb{T}}\right)$, then $\sigma(x)=\left(x_{2}, x_{3}, \ldots\right)$. By the Pimsner-Voiculescu exact sequence, there exists an exact sequence

$$
0 \rightarrow K_{1}\left(\overline{\mathcal{O}}_{\Gamma}\right) \rightarrow K_{0}\left(\overline{\mathcal{O}}_{\Gamma}^{\mathbb{T}}\right) \rightarrow K_{0}\left(\overline{\mathcal{O}}_{\Gamma}^{\mathbb{T}}\right) \rightarrow K_{0}\left(\overline{\mathcal{O}}_{\Gamma}\right) \rightarrow 0
$$

It therefore follows that $K_{0}\left(\overline{\mathcal{O}}_{\Gamma}\right)=K_{0}\left(\overline{\mathcal{O}}_{\Gamma}^{\mathbb{T}}\right) /(1-\sigma) K_{0}\left(\overline{\mathcal{O}}_{\Gamma}^{\mathbb{T}}\right)$ and $K_{1}\left(\overline{\mathcal{O}}_{\Gamma}\right)=$ $\operatorname{ker}(1-\sigma)$ on $K_{0}\left(\overline{\mathcal{O}}_{\Gamma}^{\mathbb{T}}\right)$.

Finally we consider some simple examples. First let $\Gamma=S L(2, \mathbb{Z})=$ $\mathbb{Z}_{4} * \mathbb{Z}_{2} \mathbb{Z}_{6}$. Let $\chi_{1}$ be the unit character of $\mathbb{Z}_{2}$ and let $\chi_{2}$ be the character such that $\chi_{2}(a)=-1$ where $a$ is a generator of $\mathbb{Z}_{2}$. These are one-dimensional and exhaust all the irreducible characters. Then we have the corresponding matrix

$$
A_{\Gamma}=\left(\begin{array}{cccc}
0 & 0 & 1 & 0 \\
0 & 0 & 0 & 1 \\
2 & 0 & 0 & 0 \\
0 & 2 & 0 & 0
\end{array}\right)
$$

Hence the corresponding $K$-groups are $K_{0}\left(\mathcal{O}_{\Gamma}\right)=0$ and $K_{1}\left(\mathcal{O}_{\Gamma}\right)=0$. In fact, $\mathcal{O}_{\mathbb{Z}_{4} * \mathbb{Z}_{2}} \mathbb{Z}_{6} \simeq \mathcal{O}_{\mathbb{Z}_{2} * \mathbb{Z}_{3}} \oplus \mathcal{O}_{\mathbb{Z}_{2} * \mathbb{Z}_{3}} \simeq \mathcal{O}_{2} \oplus \mathcal{O}_{2}$ 
Next let $\Gamma=\mathfrak{S}_{4} * \mathfrak{S}_{3} \mathfrak{S}_{4}, \tau=(12)$ and $\sigma=(123)$. Note that $\mathfrak{S}_{3}=\langle 1, \tau, \sigma\rangle$. $\mathfrak{S}_{3}$ has three irreducible characters:

\begin{tabular}{|c|c|c|c|}
\hline & 1 & $\tau$ & $\sigma$ \\
\hline$\chi_{1}$ & 1 & 1 & 1 \\
\hline$\chi_{2}$ & 1 & -1 & 1 \\
\hline$\chi_{3}$ & 2 & 0 & -1 \\
\hline
\end{tabular}

Moreover, $\mathfrak{S}_{3} \backslash \mathfrak{S}_{4} / \mathfrak{S}_{3}$ has only two points; say $\mathfrak{S}_{3}$ and $\mathfrak{S}_{3} x \mathfrak{S}_{3}$ with $x=$ (12)(34). Then we obtain the corresponding matrix

$$
A_{\Gamma}=\left(\begin{array}{cccccc}
0 & 0 & 0 & 1 & 0 & 1 \\
0 & 0 & 0 & 0 & 1 & 1 \\
0 & 0 & 0 & 1 & 1 & 2 \\
1 & 0 & 1 & 0 & 0 & 0 \\
0 & 1 & 1 & 0 & 0 & 0 \\
1 & 1 & 2 & 0 & 0 & 0
\end{array}\right) .
$$

Hence this gives $K_{0}\left(\mathcal{O}_{\Gamma}\right)=\mathbb{Z} \oplus \mathbb{Z}_{4}$ and $K_{1}\left(\mathcal{O}_{\Gamma}\right)=\mathbb{Z}$. In this case, $\Gamma$ satisfies the condition of Theorem 6.3. So $\mathcal{O}_{\Gamma}$ is a simple, nuclear, purely infinite $C^{*}$ algebra.

\section{$\S 8 . \quad$ KMS States on $\mathcal{O}_{\Gamma}$}

In this section, we investigate the relationship between KMS states on $\mathcal{O}_{\Gamma}$ for generalized gauge actions and random walks on $\Gamma$. Throughout this section, we assume that all groups $G_{i}$ are finite though we can carry out the same arguments if $G_{i}=\mathbb{Z} \times H$ for some $i \in I$. Let $\omega=\left(\omega_{i}\right)_{i \in I} \in \mathbb{R}_{+}^{|I|}$. By the universality of $\mathcal{O}_{\Gamma}$, we can define an automorphism $\alpha_{t}^{\omega}$ for any $t \in \mathbb{R}$ on $\mathcal{O}_{\Gamma}$ by $\alpha_{t}^{\omega}\left(S_{g}\right)=e^{\sqrt{-1} \omega_{i} t} S_{g}$ for $g \in G_{i} \backslash H$ and $\alpha_{t}^{\omega}\left(U_{h}\right)=U_{h}$ for $h \in H$. Hence we obtain the $\mathbb{R}$-action $\alpha^{\omega}$ on $\mathcal{O}_{\Gamma}$. We call it the generalized gauge action with respect to $\omega$. We will only consider actions of these types and determine KMS states on $\mathcal{O}_{\Gamma}$ for these actions.

In [32], Woess showed that our boundary $\Omega$ can be identified with the Poisson boundary of random walks satisfying certain conditions. The reader is referred to [33] for a good book of random walks.

Let $\mu$ be a probability measure on $\Gamma$ and consider a random walk governed by $\mu$, i.e. the transition probability from $x$ to $y$ given by

$$
p(x, y)=\mu\left(x^{-1} y\right) .
$$


A random walk is said to be irreducible if for any $x, y \in \Gamma, p^{(n)}(x, y) \neq 0$ for some integer $n$, where

$$
p^{(n)}(x, y)=\sum_{x_{1}, x_{2}, \ldots, x_{n-1} \in \Gamma} p\left(x, x_{1}\right) p\left(x_{1}, x_{2}\right) \cdots p\left(x_{n-1}, y\right) .
$$

A probability measure $\nu$ on $\Omega$ is said to be stationary with respect to $\mu$ if $\nu=\mu * \nu$, where $\mu * \nu$ is defined by

$$
\int_{\Omega} f(\omega) d \mu * \nu(\omega)=\int_{\Omega} \int_{\operatorname{supp} \mu} f(g \omega) d \mu(g) d \nu(\omega), \quad \text { for } \quad f \in C(\Omega, \nu) .
$$

By [32, Theorem 9.1], if a random walk governed by a probability measure $\mu$ on $\Gamma$ is irreducible, then there exists a unique stationary probability measure $\nu$ on $\Omega$ with respect to $\mu$. Moreover if $\mu$ has finite support, then the Poisson boundary coincides with $(\Omega, \nu)$.

If $\nu$ is a probability measure on the compact space $\Omega$, then we can define a state $\phi_{\nu}$ by

$$
\phi_{\nu}(X)=\int_{\Omega} E(X) d \nu \quad \text { for } \quad X \in \mathcal{O}_{\Gamma},
$$

where $E$ is the canonical conditional expectation of $C(\Omega) \rtimes_{r} \Gamma$ onto $C(\Omega)$.

One of our purposes in this section is to prove that there exists a random walk governed by a probability measure $\mu$ that induces the stationary measure $\nu$ on $\Omega$ such that the corresponding state $\phi_{\nu}$ is the unique KMS state for $\alpha^{\omega}$. Namely,

Theorem 8.1. $\quad$ Assume that the matrix $A_{\Gamma}$ obtained in the preceding section is irreducible. For any $\omega=\left(\omega_{i}\right)_{i \in I} \in \mathbb{R}_{+}^{|I|}$, there exists a unique probability measure $\mu$ with the following properties:

(i) $\operatorname{supp}(\mu)=\bigcup_{i \in I} G_{i} \backslash H$.

(ii) $\mu(g h)=\mu(g)$ for any $g \in \bigcup_{i \in I} G_{i} \backslash H$ and $h \in H$.

(iii) The corresponding unique stationary measure $\nu$ on $\Omega$ induces the unique KMS state $\phi_{\nu}$ for $\alpha^{\omega}$ and the corresponding inverse temperature $\beta$ is also unique.

We need the hypothesis of the irreducibility of the matrix $A_{\Gamma}$ for the uniqueness of the KMS state. Though it is, in general, difficult to check the irreducibility of $A_{\Gamma}$, by Theorem 6.5 , the condition of simplicity of $\mathcal{O}_{\Gamma}$ in Corollary 6.4 is also a sufficient condition for irreducibility of $A_{\Gamma}$. To obtain the theorem, we first present two lemmas. 
Lemma 8.2. Assume that $\nu$ is a probability measure on $\Omega$. Then the corresponding state $\phi_{\nu}$ is the KMS state for $\alpha^{\omega}$ if and only if $\nu$ satisfies the following conditions:

$$
\nu\left(\Omega\left(x_{1} \cdots x_{m}\right)\right)=\frac{e^{-\beta \omega_{i_{1}}} \cdots e^{-\beta \omega_{i_{m-1}}}}{\left[G_{i_{m}}: H\right]-1+e^{\beta \omega_{i_{m}}}},
$$

for $x_{k} \in \Omega_{i_{k}}$ with $i_{1} \neq \cdots \neq i_{m}$, where $\Omega\left(x_{1} \cdots x_{m}\right)$ is the cylinder subset of $\Omega$ defined by

$$
\Omega\left(x_{1} \cdots x_{m}\right)=\left\{(x(n))_{n \geq 1} \in \Omega \mid x(1)=x_{1}, \ldots, x(m)=x_{m}\right\} .
$$

Proof. $\phi_{\nu}$ is the KMS state for $\alpha^{\omega}$ if and only if

$$
\phi_{\nu}\left(S_{\xi} P_{i} U_{h} S_{\eta}^{*} \cdot S_{\sigma} P_{j} U_{k} S_{\tau}^{*}\right)=\phi\left(S_{\sigma} P_{j} U_{k} S_{\tau}^{*} \cdot \alpha_{\sqrt{-1} \beta}^{\omega}\left(S_{\xi} P_{i} U_{h} S_{\eta}^{*}\right)\right)
$$

for any $\xi, \eta, \sigma, \tau \in \Delta, h, k \in H$ and $i, j \in I$.

We may assume that $|\xi|+|\sigma|=|\eta|+|\tau|$ and $|\eta| \geq|\sigma|$. Set $|\xi|=p,|\eta|=$ $q,|\sigma|=s,|\tau|=t$ and let $\xi=\xi_{1} \cdots \xi_{p}, \eta=\eta_{1} \cdots \eta_{q}$ with $\xi_{k} \in \Omega_{i_{k}} \backslash\{e\}, \eta_{l} \in$ $\Omega_{j_{l}} \backslash\{e\}$ and $i_{1} \neq \cdots \neq i_{p}, j_{1} \neq \cdots \neq j_{q}$. Then

$$
\begin{aligned}
\phi_{\nu}\left(S_{\xi} P_{i} U_{h} S_{\eta}^{*} \cdot S_{\sigma} P_{j} U_{k} S_{\tau}^{*}\right) & =\delta_{\eta_{1} \cdots \eta_{s}, \sigma} \delta_{\eta_{s+1}, j} \phi_{\nu}\left(S_{\xi} P_{i} U_{h} S_{\eta_{s+1} \cdots \eta_{q}}^{*} U_{k} S_{\tau}^{*}\right) \\
& =\delta_{\eta_{1} \cdots \eta_{s}, \sigma} \delta_{\eta_{s+1}, j} \phi_{\nu}\left(S_{\xi h} P_{i} S_{\tau k^{-1} \eta_{s+1} \cdots \eta_{q}}\right) \\
& =\delta_{\eta_{1} \cdots \eta_{s}, \sigma} \delta_{\eta_{s+1}, j} \delta_{\xi h, \tau k^{-1} \eta_{s+1} \cdots \eta_{q}} \sum_{x \in \Omega_{i} \backslash\{e\}} \nu(\Omega(\xi x)),
\end{aligned}
$$

and

$$
\begin{aligned}
& \phi_{\nu}\left(S_{\sigma} P_{j} U_{k} S_{\tau}^{*} \cdot \alpha_{\sqrt{-1} \beta}^{\omega}\left(S_{\xi} P_{i} U_{h} S_{\eta}^{*}\right)\right) \\
& =e^{-\beta \omega_{i_{1}}} \cdots e^{-\beta \omega_{i_{p}}} e^{\beta \omega_{j_{1}}} \cdots e^{\beta \omega_{j_{q}}} \phi_{\nu}\left(S_{\sigma} P_{j} U_{k} S_{\tau}^{*} \cdot S_{\xi} P_{i} U_{h} S_{\eta}^{*}\right) \\
& =e^{-\beta \omega_{i_{1}}} \cdots e^{-\beta \omega_{i_{p}}} e^{\beta \omega_{j_{1}}} \cdots e^{\beta \omega_{j_{q}}} \delta_{\tau, \xi_{1} \cdots \xi_{t}} \delta_{\xi_{t+1}, j} \phi_{\nu}\left(S_{\sigma k \xi_{t+1} \cdots \xi_{p} h} P_{i} S_{\eta}^{*}\right) \\
& =e^{-\beta \omega_{i_{1}}} \cdots e^{-\beta \omega_{i_{p}}} e^{\beta \omega_{j_{1}}} \cdots e^{\beta \omega_{j_{q}}} \delta_{\tau, \xi_{1} \cdots \xi_{t}} \delta_{\xi_{t+1}, j} \delta_{\sigma k \xi_{t+1} \cdots \xi_{p} h, \eta} \sum_{x \in \Omega_{i} \backslash\{e\}} \nu(\Omega(\eta x)),
\end{aligned}
$$

where $\delta_{g, i}=1$ only if $g \in G_{i} \backslash H$. Therefore the corresponding state $\phi_{\nu}$ is the KMS state for $\alpha^{\omega}$ if and only if $\nu$ satisfies the following conditions:

$$
\nu\left(\Omega\left(\xi_{1} \ldots \xi_{p} x\right)\right)=e^{-\beta \omega_{i_{1}}} \cdots e^{-\beta \omega_{i_{p}}} \nu(\Omega(x)),
$$

for $x \in \Omega_{i} \backslash\{e\}$ with $i \neq i_{p}$. 
Now we assume that $\phi_{\nu}$ is the KMS state for $\alpha^{\omega}$. Then for $i \in I$,

$$
\begin{aligned}
\nu\left(Y_{i}\right) & =\phi_{\nu}\left(P_{i}\right) \\
& =\sum_{g \in \Omega_{i} \backslash\{e\}} \phi_{\nu}\left(S_{g} S_{g}^{*}\right) \\
& =\sum_{g \in \Omega_{i} \backslash\{e\}} \phi_{\nu}\left(S_{g}^{*} \alpha_{\sqrt{-1}^{\prime}}^{\omega}\left(S_{g}\right)\right) \\
& =e^{-\beta \omega_{i}} \sum_{g \in \Omega_{i} \backslash\{e\}} \phi_{\nu}\left(Q_{g}\right) \\
& =e^{-\beta \omega_{i}} \sum_{g \in \Omega_{i} \backslash\{e\}} \phi_{\nu}\left(1-P_{i}\right) \\
& =e^{-\beta \omega_{i}}\left(\left[G_{i}: H\right]-1\right)\left(1-\nu\left(Y_{i}\right)\right) .
\end{aligned}
$$

Hence,

$$
\nu\left(Y_{i}\right)=\frac{\left[G_{i}: H\right]-1}{\left[G_{i}: H\right]-1+e^{\beta \omega_{i}}} .
$$

Moreover,

$$
\begin{aligned}
\nu\left(\Omega\left(x_{1} \ldots x_{m}\right)\right) & =\phi_{\nu}\left(S_{x_{1}} \cdots S_{x_{m}} S_{x_{m}}^{*} \cdots S_{x_{1}}^{*}\right) \\
& =\phi_{\nu}\left(S_{x_{m}}^{*} \cdots S_{x_{1}}^{*} \alpha_{\sqrt{-1}^{\prime}}^{\omega}\left(S_{x_{1}} \cdots S_{x_{m}}\right)\right) \\
& =e^{-\beta \omega_{i_{1}}} \cdots e^{-\beta \omega_{i_{m}}} \phi_{\nu}\left(Q_{x_{m}}\right) \\
& =e^{-\beta \omega_{i_{1}}} \cdots e^{-\beta \omega_{i_{m}}}\left(1-\nu\left(\Omega\left(Y_{i_{m}}\right)\right)\right) \\
& =\frac{e^{-\beta \omega_{i_{1}}} \cdots e^{-\beta \omega_{i_{m}-1}}}{\left[G_{i_{m}}: H\right]-1+e^{\beta \omega_{i_{m}}}} .
\end{aligned}
$$

Conversely, suppose that a probability measure $\nu$ satisfies the condition of this lemma. By the first part of this proof, $\phi_{\nu}$ is the KMS state for $\alpha^{\omega}$.

Lemma 8.3. Assume that $\nu$ is the unique stationary measure on $\Omega$ with respect to a random walk on $\Gamma$, governed by a probability measure $\mu$ with the conditions (i), (ii) in Theorem 8.1. Then $\phi_{\nu}$ is a $\beta$-KMS state for $\alpha^{\omega}$ if and only if $\mu$ satisfies the following conditions:

$$
\mu(g)=\frac{\prod_{j \neq i} C_{j}}{\sum_{k \in I}\left(g_{k} \prod_{l \neq k} C_{l}\right)} \quad \text { for } \quad g \in G_{i} \backslash H \quad \text { and } \quad i \in I,
$$

where $g_{i}=\left|G_{i} \backslash H\right|$ and $C_{i}=\left(1-e^{-\beta \omega_{i}}\right) g_{i}-\left(1-e^{\beta \omega_{i}}\right)|H|$ for $i \in I$. 
Proof. Assume that $\phi_{\nu}$ is a $\beta$-KMS state for $\alpha^{\omega}$. For any $f \in C(\Omega)$,

$$
\begin{aligned}
\iint f(\omega) d \nu(\omega) & =\iint f(\omega) d \mu * \nu(\omega) \\
& =\iint f(g \omega) d \nu(\omega) d \mu(g) \\
& =\iint\left(\lambda_{g}^{*} f \lambda_{g}\right)(\omega) d \nu(\omega) d \mu(g) \\
& =\sum_{g \in \operatorname{supp}(\mu)} \mu(g) \phi_{\nu}\left(\lambda_{g}^{*} f \lambda_{g}\right) \\
& =\sum_{g \in \operatorname{supp}(\mu)} \mu(g) \phi_{\nu}\left(f \lambda_{g} \alpha_{\sqrt{-1} \beta}^{\omega}\left(\lambda_{g}^{*}\right)\right),
\end{aligned}
$$

where $\mathcal{O}_{\Gamma} \simeq C(\Omega) \rtimes_{r} \Gamma=C^{*}\left(f, \lambda_{\gamma} \mid f \in C(\Omega), \gamma \in \Gamma\right)$.

Put $f=\chi_{\Omega(x)}=P_{x}$ for $i \in I$ and $x \in \Omega_{i} \backslash\{e\}$. Since $\lambda_{g}=S_{g}+$ $\sum_{g^{\prime} \in \Omega_{i^{\prime}} \backslash H \cup g^{-1} H} S_{g g^{\prime}} S_{g^{\prime}}^{*}+S_{g^{-1}}^{*}$ for $g \in G_{i^{\prime}} \backslash H$ and $i^{\prime} \in I$, we have

$$
1=\sum_{g H=x H} \mu(g) e^{\beta \omega_{i}}+\sum_{g \in G_{i} \backslash H, g H \neq x H} \mu(g)+\sum_{g \in G_{j} \backslash H, j \neq i} \mu(g) e^{-\beta \omega_{j}}
$$

for any $i \in I$ and $x \in \Omega_{i} \backslash\{e\}$. Let $x, y \in \Omega_{i} \backslash\{e\}$ with $x H \neq y H$. Then

$$
\begin{aligned}
& 1=\sum_{g H=x H} \mu(g) e^{\beta \omega_{i}}+\sum_{g H \neq x H} \mu(g)+\sum_{g \in G_{j} \backslash H, j \neq i} \mu(g) e^{-\beta \omega_{j}}, \\
& 1=\sum_{g H=y H} \mu(g) e^{\beta \omega_{i}}+\sum_{g H \neq y H} \mu(g)+\sum_{g \in G_{j} \backslash H, j \neq i} \mu(g) e^{-\beta \omega_{j}} .
\end{aligned}
$$

By the above equations, we have $\mu(x)=\mu(y)$, and then it follows from hypothesis (ii) in Theorem 8.1 that $\mu(g)=\mu_{i}$ for any $g \in G_{i} \backslash H$. Therefore we have

$$
1=|H| e^{\beta \omega_{i}} \mu_{i}+\left(g_{i}-|H|\right) \mu_{i}+\sum_{j \neq i} g_{j} e^{-\beta \omega_{j}} \mu_{j}
$$

for any $i \in I$, where $g_{i}=\left|G_{i} \backslash H\right|$. Thus by considering the above equations for $i$ and $j \in I$,

$$
|H| e^{\beta \omega_{i}} \mu_{i}-|H| e^{\beta \omega_{j}} \mu_{j}+\left(g_{i}-|H|\right) \mu_{i}-\left(g_{j}-|H|\right) \mu_{j}+g_{j} e^{-\beta \omega_{j}} \mu_{j}-g_{i} e^{-\beta \omega_{i}} \mu_{i}=0 .
$$

Hence we obtain the equation,

$$
\left(|H| e^{\beta \omega_{i}}+g_{i}-|H|-g_{i} e^{-\beta \omega_{i}}\right) \mu_{i}=\left(|H| e^{\beta \omega_{j}}+g_{j}-|H|-g_{j} e^{-\beta \omega_{j}}\right) \mu_{j} .
$$


Since $\mu\left(\bigcup_{i \in I} G_{i} \backslash H\right)=1$, we have

$$
g_{i} \mu_{i}+\sum_{j \neq i} g_{j} \frac{\left(1-e^{-\beta \omega_{i}}\right) g_{i}-\left(1-e^{-\beta \omega_{i}}\right)|H|}{\left(1-e^{-\beta \omega_{j}}\right) g_{j}-\left(1-e^{-\beta \omega_{j}}\right)|H|} \mu_{i}=1 .
$$

We put $C_{i}=\left(1-e^{-\beta \omega_{i}}\right) g_{i}-\left(1-e^{-\beta \omega_{i}}\right)|H|$ and then

$$
\left(g_{i}+C_{i} \sum_{j \neq i} \frac{g_{j}}{C_{j}}\right) \mu_{i}=1 .
$$

Therefore

$$
\begin{aligned}
\mu_{i} & =\frac{1}{g_{i}+C_{i} \sum_{j \neq i} g_{j} / C_{j}} \\
& =\frac{\prod_{j \neq i} C_{j}}{g_{i} \prod_{j \neq i} C_{j}+\sum_{j \neq i}\left(g_{j} C_{i} \prod_{k \neq i, j} C_{k}\right)} \\
& =\frac{\prod_{j \neq i} C_{j}}{\sum_{k \in I} g_{k} \prod_{l \neq k} C_{l}} .
\end{aligned}
$$

On the other hand, let $\nu$ be the probability measure on $\Omega$ satisfying the condition in Lemma 8.2. Then the corresponding state $\phi_{\nu}$ is the KMS state. It is enough to check that $\mu * \nu=\nu$ by [32]. Since

$$
\nu\left(\Omega\left(x_{1} \cdots x_{n}\right)\right)=e^{-\beta \omega_{i_{1}}} \cdots e^{-\beta \omega_{i_{n}-1}} \nu\left(\Omega\left(x_{n}\right)\right),
$$

for $x_{k} \in \Omega_{i_{k}} \backslash\{e\}$ with $i_{1} \neq \cdots \neq i_{n}$, we have

$$
\begin{aligned}
\mu * \nu & \left.\nu\left(x_{1} \cdots x_{n}\right)\right) \\
= & \iint \chi_{\Omega\left(x_{1} \cdots x_{n}\right)}(\omega) d \mu * \nu(\omega) \\
= & \sum_{g \in \operatorname{supp} \mu} \mu(g) \int\left(\lambda_{g}^{*} \chi_{\Omega\left(x_{1} \cdots x_{n}\right)} \lambda_{g}\right)(\omega) d \nu(\omega) \\
= & \sum_{g \in G_{i_{1}} \backslash H, x_{1} H=g H} \mu_{i_{1}} \phi_{\nu}\left(S_{x_{2}} \cdots S_{x_{n}} S_{x_{n}}^{*} \cdots S_{x_{2}}^{*}\right) \\
& +\sum_{g \in G_{i_{1}} \backslash H, x_{1} H \neq g H} \mu_{i_{1}} \phi_{\nu}\left(S_{g^{-1} x_{1}} S_{x_{2}} \cdots S_{x_{n}} S_{x_{n}}^{*} \cdots S_{x_{2}}^{*} S_{g^{-1} x_{1}}^{*}\right) \\
& +\sum_{g \in G_{i} \backslash H, i \neq i_{1}} \mu_{i} \phi_{\nu}\left(S_{g^{-1}} S_{x_{1}} S_{x_{2}} \cdots S_{x_{n}} S_{x_{n}}^{*} \cdots S_{x_{2}}^{*} S_{x_{1}}^{*} S_{g^{1}}^{*}\right) \\
= & \left(|H| e^{\beta \omega_{i_{1}}} \mu_{i_{1}}+\left(g_{i_{1}}-|H|\right) \mu_{i_{1}}+\sum_{i \neq i_{1}} g_{i} e^{-\beta \omega_{i}} \mu_{i}\right) \nu\left(\Omega\left(x_{1} \cdots x_{n}\right)\right)
\end{aligned}
$$




$$
=\nu\left(\Omega\left(x_{1} \ldots x_{n}\right)\right)
$$

To prove the uniqueness of KMS states of $\mathcal{O}_{\Gamma}$, we need the irreducibility of the matrix $A_{\Gamma}$. (See [13] for KMS states on Cuntz-Krieger algebras.) Set an irreducible matrix $B=[B((i, k),(j, l))]=\left[e^{-\beta \omega_{i}} A_{\Gamma}^{t}((i, k),(j, l))\right]$. Let $K_{\beta}$ be the set of all $\beta$-KMS states for the action $\alpha^{\omega}$. We put

$$
L_{\beta}=\left\{y=[y(i, k)] \in \mathbb{R}^{N} \mid B y=y, \quad y(i, k) \geq 0, \quad \sum_{i \in I} \sum_{k=1}^{r} n_{k} y(i, k)=1\right\} .
$$

We now have the necessary ingredients for the proof of Theorem 8.1.

Proof of Theorem 8.1. We first prove the uniqueness of the corresponding inverse temperature. Let $\phi$ be a $\beta$-KMS state for $\alpha^{\omega}$. For $i \in I$,

$$
\begin{aligned}
\phi\left(P_{i}\right) & =\sum_{g \in \Omega_{i} \backslash\{e\}} \phi\left(S_{g} S_{g}^{*}\right) \\
& =\sum_{g \in \Omega_{i} \backslash\{e\}} \phi\left(S_{g}^{*} \alpha_{\sqrt{-1} \beta}^{\omega}\left(S_{g}\right)\right) \\
& =e^{-\beta \omega_{i}} \sum_{g \in \Omega_{i} \backslash\{e\}} \phi\left(Q_{g}\right) \\
& =e^{-\beta \omega_{i}}\left(\left[G_{i}: H\right]-1\right)\left(1-\phi\left(P_{i}\right)\right) .
\end{aligned}
$$

Thus $\phi\left(P_{i}\right)=\lambda_{i}(\beta) /\left(1+\lambda_{i}(\beta)\right)$, where $\lambda_{i}(\beta)=e^{-\beta \omega_{i}}\left(\left[G_{i}: H\right]-1\right)$. Since $\sum_{i \in I} P_{i}=1$,

$$
|I|-1=\sum_{i \in I} \frac{1}{1+\lambda_{i}(\beta)} .
$$

The function $\sum_{i \in I} 1 /\left(1+\lambda_{i}(\beta)\right)$ is a monotone increasing continuous function such that

$$
\sum_{i \in I} \frac{1}{1+\lambda_{i}(\beta)}=\left\{\begin{array}{cl}
\sum_{i \in I} 1 /\left[G_{i}: H\right] & \text { if } \beta=0 \\
|I| & \text { if } \beta \rightarrow \infty
\end{array}\right.
$$

Since $\sum_{i \in I} 1 /\left[G_{i}: H\right] \leq|I| / 2 \leq|I|-1$, there exists a unique $\beta$ satisfying

$$
|I|-1=\sum_{i \in I} \frac{1}{\left(\left[G_{i}: H\right]-1\right) e^{-\beta \omega_{i}}+1} .
$$

Therefore we obtain the uniqueness of the inverse temperature $\beta$. 
We will next show the uniqueness of the KMS state $\phi_{\nu}$. We claim that $K_{\beta}$ is in one-to-one correspondence with $\mathrm{七}_{\beta}$. In fact, we define a map $f$ from $K_{\beta}$ to $\mathrm{E}_{\beta}$ by

$$
f(\phi)=\left[\phi(P(i, k)) / n_{k}\right]
$$

Indeed,

$$
\begin{aligned}
e^{\beta \omega_{i}} \phi(P(i, k)) & =\sum_{g \in \Omega_{i} \backslash\{e\}} \phi\left(p_{k} S_{g} \alpha_{\sqrt{-1} \beta}^{\omega}\left(S_{g}^{*}\right)\right) \\
& =\sum_{g \in \Omega_{i} \backslash\{e\}} \phi\left(S_{g}^{*} p_{k} S_{g}\right) \\
& =\frac{n_{k}}{|H|} \sum_{g \in \Omega_{i} \backslash\{e\}} \sum_{h \in H} \overline{\chi_{k}(h)} \phi\left(S_{g}^{*} U_{h} S_{g}\right) \\
& =\frac{n_{k}}{|H|} \sum_{g \in \Omega_{i} \backslash\{e\}} \sum_{h \in H(g)} \overline{\chi_{k}(h)} \phi\left(Q_{g} U_{g^{-1} h g}\right) \\
& =\frac{n_{k}}{|H|} \sum_{g \in \Omega_{i} \backslash\{e\}} \sum_{h \in H(g)} \overline{\chi_{k}(h)} \sum_{j \neq i} \phi\left(P_{j} U_{g^{-1} h g} P_{j}\right) \\
& =\frac{n_{k}}{|H|} \sum_{g \in \Omega_{i} \backslash\{e\}} \sum_{h \in H(g)} \overline{\chi_{k}(h)} \sum_{j \neq i} \sum_{l=1}^{r} \phi\left(P(j, l) U_{g^{-1} h g} P(j, l)\right) .
\end{aligned}
$$

Since $\phi$ is a trace on $C^{*}\left(P(j, l) U_{h} P(j, l) \mid h \in H\right) \simeq M_{n_{l}}(\mathbb{C})$ and $M_{n_{l}}(\mathbb{C})$ has a unique tracial state, we have

$$
\phi\left(P(j, l) U_{g^{-1} h g} P(j, l)\right)=\chi_{l}\left(g^{-1} h g\right) \frac{\phi(P(j, l))}{n_{l}} .
$$

Therefore, by the same arguments as in the previous section, we obtain

$$
\begin{aligned}
e^{\beta \omega_{i}} \phi(P(i, k)) & =\frac{n_{k}}{|H|} \sum_{g \in \Omega_{i} \backslash\{e\}} \sum_{h \in H(g)} \overline{\chi_{k}(h)} \sum_{j \neq i} \sum_{l=1}^{r} \phi\left(P(j, l) U_{g^{-1} h g} P(j, l)\right) \\
& =n_{k} \sum_{x \in X_{i} \backslash\{e\}} \sum_{j \neq i} \sum_{l=1}^{r}\left\langle\chi_{k}, \chi_{l}^{x}\right\rangle_{H(x)} \phi(P(j, l)) / n_{l} \\
& =n_{k} \sum_{(j, l)} A_{\Gamma}((j, l),(i, k)) \phi(P(j, l)) / n_{l} .
\end{aligned}
$$

Hence this is well-defined.

Suppose that $\nu$ is the probability measure in Lemma 8.2 and $\phi_{\nu}$ is the induced $\beta$-KMS state for $\alpha^{\omega}$. Set a vector $y=\left[y(i, k)=\phi_{\nu}(P(i, k)) / n_{k}\right]$. Since $y$ is strictly positive and $B$ is irreducible, 1 is the eigenvalue which dominates 
the absolute value of all eigenvalue of $B$ by the Perron-Frobenius theorem. It also follows from the Perron-Frobenius theorem that $L_{\beta}$ has only one element. Hence $f$ is surjective.

Let $\phi \in K_{\beta}$. For $\xi=\xi_{i_{1}} \cdots \xi_{i_{n}}, \eta=\eta_{j_{1}} \cdots \eta_{j_{n}}$ with $i_{1} \neq \cdots \neq i_{n}, j_{1} \neq$ $\cdots \neq j_{n}, h \in H$ and $i \in I$,

$$
\begin{aligned}
e^{\beta \omega_{j_{1}} \cdots e^{\beta \omega_{j_{n}}} \phi\left(S_{\xi} U_{h} P_{i} S_{\eta}^{*}\right)} & =\phi\left(S_{\xi} U_{h} P_{i} \alpha_{\sqrt{-1} \beta}^{\omega}\left(S_{\eta}^{*}\right)\right) \\
& =\phi\left(S_{\eta}^{*} S_{\xi} U_{h} P_{i}\right) \\
& =\delta_{\xi, \eta} \phi\left(U_{h} P_{i}\right) \\
& =\delta_{\xi, \eta} \sum_{k=1}^{r} \phi\left(U_{h} P(i, k)\right) \\
& =\delta_{\xi, \eta} \sum_{k=1}^{r} \chi_{k}(h) \phi(P(i, k)) / n_{k},
\end{aligned}
$$

because $\phi$ is a trace on $C^{*}\left(U_{h} P(i, k) \mid h \in H\right) \simeq M_{n_{k}}(\mathbb{C})$. If $f(\phi)=f(\psi)$, then the above calculations imply $\phi=\psi$ on $\mathcal{O}_{\Gamma}^{\mathbb{T}}$. By the KMS condition, $\phi(b)=0=\psi(b)$ for $b \notin \mathcal{O}_{\Gamma}^{\mathbb{T}}$. Thus $\phi=\psi$ and $f$ is injective. Therefore $\phi_{\nu}$ is the unique $\beta$-KMS state for $\alpha^{\omega}$.

Remark. Let $\nu$ be the corresponding probability measure with the gauge action $\alpha$. Under the identification $L^{\infty}(\Omega, \nu) \rtimes_{w} \Gamma \simeq \pi_{\nu}\left(\mathcal{O}_{\Gamma}\right)^{\prime \prime}$, we can determine the type of the factor by essentially the same arguments as in [13]. If $H$ is trivial, then $\mathcal{O}_{\Gamma}$ is a Cuntz-Krieger algebra for some irreducible matrix with 0-1 entries. In this case, we can always apply the result in [13]. This fact generalizes [25]. If $H$ is not trivial, then by using the condition of simplicity of $\mathcal{O}_{\Gamma}$ in Corollary 6.4 to check the irreducibility of the matrix $A_{\Gamma}$, we can apply Theorem 8.1. In the special case where $G_{i}=G$ for all $i \in I$, we can easily determine the type of the factor $\pi_{\nu}\left(\mathcal{O}_{\Gamma}\right)^{\prime \prime}$ for the gauge action. The factor $\pi_{\nu}\left(\mathcal{O}_{\Gamma}\right)^{\prime \prime}$ is of type $\mathrm{III}_{\lambda}$ where $\lambda=1 /([G: H]-1)^{2}$ if $|I|=2$ and $\lambda=1 /(|I|-1)([G: H]-1)$ if $|I|>2$. For instance, let $\Gamma=\mathfrak{S}_{4} *_{\mathfrak{S}_{3}} \mathfrak{S}_{4}$. We have already obtained the matrix $A_{\Gamma}$ in Section 7 , but we can determine that the factor $L^{\infty}(\Omega, \nu) \rtimes_{w} \Gamma$ is of type $\mathrm{III}_{1 / 9}$ without using $A_{\Gamma}$.

We next discuss the converse. Namely any $\mathbb{R}$-actions that have KMS states induced by a probability measure $\mu$ on $\Gamma$ with some conditions is, in fact, a generalized gauge action.

Let $\mu$ be a given probability measure on $\Gamma$ with $\operatorname{supp}(\mu)=\bigcup_{i \in I} G_{i} \backslash H$. By [32], there exists an unique probability measure $\nu$ on $\Omega$ such that $\mu * \nu=\nu$. Let $\left(\pi_{\nu}, H_{\nu}, x_{\nu}\right)$ be the GNS-representation of $\mathcal{O}_{\Gamma}$ with respect to the state $\phi_{\nu}$. 
We also denote a vector state of $x_{\nu}$ by $\phi_{\nu}$.

$$
\phi_{\nu}(a)=\left\langle a x_{\nu}, x_{\nu}\right\rangle \quad \text { for } \quad a \in \pi_{\nu}\left(\mathcal{O}_{\Gamma}\right)^{\prime \prime} .
$$

Let $\sigma_{t}^{\nu}$ be the modular automorphism group of $\phi_{\nu}$.

Theorem 8.4. $\quad$ Suppose that $\mu$ is a probability measure on $\Gamma$ such that $\operatorname{supp}(\mu)=\bigcup_{i \in I} G_{i} \backslash H$ and $\mu(g)=\mu(h g)$ for any $g \in \bigcup_{i \in I} G_{i} \backslash H, h \in H$. If $\nu$ is the corresponding stationary measure with respect to $\mu$, then there exists $\omega_{g} \in \mathbb{R}_{+}$such that

$$
\sigma_{t}^{\nu}\left(\pi_{\nu}\left(S_{g}\right)\right)=e^{\sqrt{-1} \omega_{g} t} \pi_{\nu}\left(S_{g}\right) \quad \text { for } \quad g \in G_{i} \backslash H, i \in I,
$$

and

$$
\sigma_{t}^{\nu}\left(\pi_{\nu}\left(U_{h}\right)\right)=\pi_{\nu}\left(U_{h}\right) \quad \text { for } \quad h \in H .
$$

Proof. To prove that $\sigma_{t}^{\nu}\left(\pi_{\nu}\left(S_{g}\right)\right)=e^{\sqrt{-1} \omega_{g} t} \pi_{\nu}\left(S_{g}\right)$, it suffices to show that there exists $\zeta_{g} \in \mathbb{R}_{+}$such that

$$
\phi_{\nu}\left(\pi_{\nu}\left(S_{g}\right) a\right)=\zeta_{g} \phi_{\nu}\left(a \pi_{\nu}\left(S_{g}\right)\right) \quad \text { for } \quad g \in G_{i} \backslash H, a \in \pi_{\nu}\left(\mathcal{O}_{\Gamma}\right)^{\prime \prime} .
$$

In fact, Let $\Delta_{\nu}$ be the modular operator and $J_{\nu}$ be the modular conjugate of $\phi_{\nu}$.

$$
\text { (left hand side of } \begin{aligned}
(*)) & =\left\langle\pi_{\nu}\left(S_{g}\right) a x_{\nu}, x_{\nu}\right\rangle \\
& =\left\langle a x_{\nu}, \pi_{\nu}\left(S_{g}\right)^{*} x_{\nu}\right\rangle \\
& =\left\langle a x_{\nu}, J_{\nu} \Delta_{\nu}^{1 / 2} \pi_{\nu}\left(S_{g}\right) x_{\nu}\right\rangle \\
& =\left\langle\Delta_{\nu}^{1 / 2} \pi_{\nu}\left(S_{g}\right) x_{\nu}, J_{\nu} a x_{\nu}\right\rangle \\
& =\left\langle\Delta_{\nu}^{1 / 2} \pi_{\nu}\left(S_{g}\right) x_{\nu}, \Delta_{\nu}^{1 / 2} a^{*} x_{\nu}\right\rangle .
\end{aligned}
$$

and

$$
\text { (right hand side of } \begin{aligned}
(*)) & =\zeta_{g}\left\langle a \pi_{\nu}\left(S_{g}\right) x_{\nu}, x_{\nu}\right\rangle \\
& =\zeta_{g}\left\langle\pi_{\nu}\left(S_{g}\right) x_{\nu}, a^{*} x_{\nu}\right\rangle .
\end{aligned}
$$

Therefore for $a \in \pi_{\nu}\left(\mathcal{O}_{\Gamma}\right)^{\prime \prime}$,

$$
\left\langle\Delta_{\nu}^{1 / 2} \pi_{\nu}\left(S_{g}\right) x_{\nu}, \Delta_{\nu}^{1 / 2} a^{*} x_{\nu}\right\rangle=\zeta_{g}\left\langle\pi_{\nu}\left(S_{g}\right) x_{\nu}, a^{*} x_{\nu}\right\rangle .
$$

and hence for $y \in \operatorname{dom}\left(\Delta_{\nu}^{1 / 2}\right)$, we have

$$
\left\langle\Delta_{\nu}^{1 / 2} \pi_{\nu}\left(S_{g}\right) x_{\nu}, \Delta_{\nu}^{1 / 2} y\right\rangle=\zeta_{g}\left\langle\pi_{\nu}\left(S_{g}\right) x_{\nu}, y\right\rangle
$$


Thus $\Delta_{\nu}^{1 / 2} \pi_{\nu}\left(S_{g}\right) x_{\nu} \in \operatorname{dom}\left(\Delta_{\nu}^{1 / 2}\right)$ and we obtain

$$
\Delta_{\nu} \pi_{\nu}\left(S_{g}\right) x_{\nu}=\zeta_{g} \pi_{\nu}\left(S_{g}\right) x_{\nu}
$$

Therefore

$$
\Delta_{\nu}^{\sqrt{-1} t} \pi_{\nu}\left(S_{g}\right) x_{\nu}=\zeta_{g}^{\sqrt{-1} t} \pi_{\nu}\left(S_{g}\right) x_{\nu},
$$

and then

$$
\left(\sigma_{t}^{\nu}\left(\pi_{\nu}\left(S_{g}\right)\right)-\zeta_{g}^{\sqrt{-1} t} \pi_{\nu}\left(S_{g}\right)\right) x_{\nu}=0
$$

where $\sigma_{t}^{\nu}$ is the modular automorphism group of $\phi_{\nu}$. Since $x_{\nu}$ is a separating vector,

$$
\sigma_{t}^{\nu}\left(\pi_{\nu}\left(S_{g}\right)\right)=\zeta_{g}^{\sqrt{-1} t} \pi_{\nu}\left(S_{g}\right) .
$$

Now we will show that

$$
\phi_{\nu}\left(\pi_{\nu}\left(S_{g}\right) a\right)=\zeta_{g} \phi_{\nu}\left(a \pi_{\nu}\left(S_{g}\right)\right) \quad \text { for } \quad g \in G_{i} \backslash H, a \in \pi_{\nu}\left(\mathcal{O}_{\Gamma}\right)^{\prime \prime} .
$$

We may assume that $a=f \lambda_{g^{-1}}$ for $f \in C(\Omega)$. Recall that $S_{g}=\lambda_{g} \chi_{\Omega \backslash Y_{i}} \in$ $C(\Omega) \rtimes_{r} \Gamma$. Since

$$
\phi_{\nu}\left(\pi_{\nu}\left(S_{g} a\right)\right)=\int_{\Omega \backslash Y_{i}} f\left(g^{-1} \omega\right) d \nu(\omega)=\int_{\Omega \backslash Y_{i}} f(\omega) \frac{d g^{-1} \nu}{d \nu}(\omega) d \nu(\omega),
$$

we claim that

$$
\frac{d g^{-1} \nu}{d \nu}(\omega)=\zeta_{g} \quad \text { on } \quad \Omega \backslash Y_{i} .
$$

This is the Martin kernel $K\left(g^{-1}, \omega\right)$, (See [32]). Hence it suffices to show that $K\left(g^{-1}, x\right)$ is constant for any $x=x_{1} \cdots x_{n} \in \Gamma$ such that $x_{1} \notin G_{i}$. By [32], we have

$$
K\left(g^{-1}, x\right)=\frac{G\left(g^{-1}, x\right)}{G(e, x)},
$$

where $G(y, z)=\sum_{k=1}^{\infty} p^{(k)}(y, z)$ is the Green kernel. Since any probability from $g^{-1}$ to $x$ must be through elements of $H$ at least once, we have

$$
G\left(g^{-1}, x\right)=\sum_{h \in H} F\left(g^{-1}, h\right) G(h, x),
$$

where $s^{x}=\inf \left\{n \geq 0 \mid Z_{n}=x\right\}$ and $F(g, x)=\sum_{n=0}^{\infty} \operatorname{Pr}_{g}\left[s^{x}=n\right]$ in [33]. By hypothesis $\mu(g)=\mu(h g)$ for any $g \in \bigcup_{i \in I} G_{i} \backslash H$ and $h \in H$, we have

$$
G(h, x)=G(e, x) \quad \text { for any } \quad h \in H .
$$

Therefore we have $\omega_{g}=\log \left(\sum_{h \in H} F\left(g^{-1}, h\right)\right) . \quad \sigma_{t}^{\nu}\left(\pi_{\nu}\left(U_{h}\right)\right)=\pi_{\nu}\left(U_{h}\right)$ can be proved in the same way. Hence we are done. 


\section{§9. Appendix}

Trees. We first review trees based on [15]. A graph is a pair $(V, E)$ consisting of a set of vertices $V$ and a family $E$ of two-element subsets of $V$, called edges. A path is a finite sequence $\left\{x_{1}, \ldots, x_{n}\right\} \subseteq V$ such that $\left\{x_{i}, x_{i+1}\right\} \in E$. $(V, E)$ is said to be connected if for $x, y \in V$ there exists a path $\left\{x_{1}, \ldots, x_{n}\right\}$ with $x_{1}=x, x_{n}=y$. If $(V, E)$ is a tree, then for $x, y \in V$ there exists a unique path $\left\{x_{1}, \ldots, x_{n}\right\}$ joining $x$ to $y$ such that $x_{i} \neq x_{i+2}$. We denote this path by $[x, y]$. A tree is said to be locally finite if every vertex belongs to finitely many edges. The number of edges to which a vertex of a locally finite tree belongs is called a degree. If the degree is independent of the choice of vertices, then the tree is called homogeneous.

We introduce trees for amalgamated free product groups based on [27]. Let $\left(G_{i}\right)_{i \in I}$ be a family of groups with an index set $I$. When $H$ is a group and every $G_{i}$ contains $H$ as a subgroup, then we denote ${ }^{*}{ }_{H} G_{i}$ by $\Gamma$, which is the amalgamated free product of the groups. If we choose sets $\Omega_{i}$ of left representatives of $G_{i} / H$ with $e \in \Omega_{i}$ for any $i \in I$, then each $\gamma \in \Gamma$ can be written uniquely as

$$
\gamma=g_{1} g_{2} \cdots g_{n} h
$$

where $h \in H, g_{1} \in \Omega_{i_{1}} \backslash\{e\}, \ldots, g_{n} \in \Omega_{i_{n}} \backslash\{e\}$ and $i_{1} \neq i_{2}, i_{2} \neq i_{3}, \ldots, i_{n-1} \neq$ $i_{n}$.

Now we construct the corresponding tree. At first, we assume that $I=$ $\{1,2\}$. Let

$$
V=\Gamma / G_{1} \coprod \Gamma / G_{2} \text { and } E=\Gamma / H,
$$

and the original and terminal maps $o: \Gamma / H \rightarrow \Gamma / G_{1}$ and $t: \Gamma / H \rightarrow \Gamma / G_{2}$ are natural surjections. It is easy to see that $G_{T}=(V, E)$ is a tree. In general, we assume that the element 0 does not belong to $I$. Let $G_{0}=H$ and $H_{i}=H$ for $i \in I$. Then we define

$$
V=\coprod_{i \in I \cup\{0\}} \Gamma / G_{i} \text { and } E=\coprod_{i \in I} \Gamma / H_{i}
$$

Now we define two maps $o, t: E \rightarrow V$. For $H_{i} \in E$, let

$$
o\left(H_{i}\right)=G_{0} \quad \text { and } \quad t\left(H_{i}\right)=G_{i} .
$$

For any $\gamma H_{i} \in E$, we may assume that $\gamma H=g_{1} \cdots g_{n} H_{i}$ such that $g_{k} \in \Omega_{i_{k}}$ with $i_{1} \neq \cdots \neq i_{n}$. If $i=i_{n}$ we define

$$
o\left(\gamma H_{i}\right)=\gamma G_{i_{n}} \text { and } t\left(\gamma H_{i}\right)=\gamma G_{0} .
$$


If $i \neq i_{n}$ we define

$$
o\left(\gamma H_{i}\right)=\gamma G_{0} \quad \text { and } \quad t\left(\gamma H_{i}\right)=\gamma G_{i} .
$$

Then we have a tree $G_{T}=(V, E)$.

For a tree $(V, E)$, the set $V$ is naturally a metric space. The distance $d(x, y)$ is defined by the number of edges in the unique path $[x, y]$. An infinite chain is an infinite path $\left\{x_{1}, x_{2}, \ldots\right\}$ such that $x_{i} \neq x_{i+2}$. We define an equivalence relation on the set of infinite chains. Two infinite chains $\left\{x_{1}, x_{2}, \ldots\right\},\left\{y_{1}, y_{2}, \ldots\right\}$ are equivalent if there exists an integer $k$ such that $x_{n}=y_{n+k}$ for a sufficiently large $n$. The boundary $\Omega$ of a tree is the set of the equivalence classes of infinite chains. The boundary may be thought of as a point at infinity. Next we introduce the topology into the space $V \cup \Omega$ such that $V \cup \Omega$ is compact, the points of $V$ are open and $V$ is dense in $V \cup \Omega$. It suffices to define a basis of neighborhoods for each $\omega \in \Omega$. Let $x$ be a vertex. Let $\left\{x, x_{1}, x_{2}, \ldots\right\}$ be an infinite chain representing $\omega$. For each $y=x_{n}$, the neighborhood of $\omega$ is defined to consist of all vertices and all boundary points of the infinite chains which include $[x, y]$.

Hyperbolic groups. We introduce hyperbolic groups defined by Gromov. See [18] for details. Suppose that $(X, d)$ is a metric space. We define a product by

$$
\langle x \mid y\rangle_{z}=\frac{1}{2}\{d(x, z)+d(y, z)-d(x, y)\},
$$

for $x, y, z \in X$. This is called the Gromov product. Let $\delta \geq 0$ and $w \in X$. A metric space $X$ is said to be $\delta$-hyperbolic with respect to $w$ if for $x, y, z \in X$,

$$
\langle x \mid y\rangle_{w} \geq \min \left\{\langle x \mid z\rangle_{w},\langle y \mid z\rangle_{w}\right\}-\delta .
$$

Note that if $X$ is $\delta$-hyperbolic with respect to $w$, then $X$ is $\delta$-hyperbolic with respect to any $w^{\prime} \in X$.

Definition 9.1. The space $X$ is said to be hyperbolic if $X$ is $\delta$-hyperbolic with respect to some $w \in X$ and some $\delta \geq 0$.

Suppose that $\Gamma$ is a group generated by a finite subset $S$ such that $S^{-1}=S$. Let $G(\Gamma, S)$ be the Cayley graph. The graph $G(\Gamma, S)$ has a natural word metric. Hence $G(\Gamma, S)$ is a metric space.

Definition 9.2. $\quad$ A finitely generated group $\Gamma$ is said to be hyperbolic with respect to a finite generator system $S$ if the corresponding Cayley graph $G(\Gamma, S)$ is hyperbolic with respect to the word metric. 
In fact, hyperbolicity is independent of the choice of $S$. Therefore we say that $\Gamma$ is a hyperbolic group, for short.

We define the hyperbolic boundary of a hyperbolic space $X$. Let $w \in X$ be a point. A sequence $\left(x_{n}\right)$ in $X$ is said to converge to infinity if $\left\langle x_{n} \mid x_{m}\right\rangle_{w} \rightarrow$ $\infty,(n, m \rightarrow \infty)$. Note that this is independent of the choice of $w$. The set $X_{\infty}$ is the set of all sequences converging to infinity in $X$. Then we define an equivalence relation in $X_{\infty}$. Two sequences $\left(x_{n}\right),\left(y_{n}\right)$ are equivalent if $\left\langle x_{n} \mid y_{n}\right\rangle_{w} \rightarrow \infty,(n \rightarrow \infty)$. Although this is not an equivalence relation in general, the hyperbolicity assures that it is indeed an equivalence relation. The set of all equivalent classes of $X_{\infty}$ is called the hyperbolic boundary (at infinity) and denoted by $\partial X$. Next we define the Gromov product on $X \cup \partial X$. For $x, y \in X \cup \partial X$, we choose sequences $\left(x_{n}\right),\left(y_{n}\right)$ converging to $x, y$, respectively. Then we define $\langle x \mid y\rangle=\liminf _{n \rightarrow \infty}\left\langle x_{n} \mid y_{n}\right\rangle_{w}$. Note that this is well-defined and if $x, y \in X$ then the above product coincides with the Gromov product on $X$.

Definition 9.3. The topology of $X \cup \partial X$ is defined by the following neighborhood basis:

$$
\begin{gathered}
\{y \in X \mid d(x, y)<r\} \quad \text { for } x \in X, r>0, \\
\{y \in X \cup \partial X \mid\langle x \mid y\rangle>r\} \quad \text { for } x \in \partial X, r>0 .
\end{gathered}
$$

We remark that if $X$ is a tree, then the hyperbolic boundary $\partial X$ coincides with the natural boundary $\Omega$ in the sense of [16].

Finally we prove that an amalgamated free product $\Gamma=*_{H} G_{i}$, considered in this paper, is a hyperbolic group.

Lemma 9.4. The group $\Gamma={ }^{H} G_{i}$ is a hyperbolic group.

Proof. Let $S=\left\{g \in \bigcup_{i} G_{i}|| g \mid \leq 1\right\}$. Let $G(\Gamma, S)$ be the corresponding Cayley graph. It suffices to show $(\ddagger)$ for $w=e$. For $x, y, z \in \Gamma$, we can write uniquely as follows:

$$
\begin{aligned}
& x=x_{1} \cdots x_{n} h_{x}, \\
& y=y_{1} \cdots y_{m} h_{y}, \\
& z=z_{1} \cdots z_{k} h_{z},
\end{aligned}
$$

where

$$
\begin{aligned}
& x_{1} \in \Omega_{i\left(x_{1}\right)}, \ldots, x_{n} \in \Omega_{i\left(x_{n}\right)}, h_{x} \in H, \\
& y_{1} \in \Omega_{i\left(y_{1}\right)}, \ldots, y_{m} \in \Omega_{i\left(y_{m}\right)}, h_{y} \in H, \\
& z_{1} \in \Omega_{i\left(z_{1}\right)}, \ldots, z_{k} \in \Omega_{i\left(z_{k}\right)}, h_{z} \in H .
\end{aligned}
$$


such that each element has length one. Then $d(x, e)=n, d(y, e)=m$ and $d(z, e)=k$. If $i\left(x_{1}\right)=i\left(y_{1}\right), \ldots, i\left(x_{l(x, y)}\right)=i\left(y_{l(x, y)}\right)$ and $i\left(x_{l(x, y)+1}\right) \neq$ $i\left(y_{l(x, y)+1}\right)$, then $\langle x \mid y\rangle_{e}=l(x, y)$. Similarly, we obtain the positive integers $l(x, z), l(y, x)$ such that $\langle x \mid z\rangle_{e}=l(x, z),\langle y \mid z\rangle_{e}=l(y, z)$. We can have ( $\left.\ddagger\right)$ with $\delta=0$.

\section{Acknowledgement}

The author gives special thanks to Professor Masaki Izumi for various comments and many important suggestions.

\section{References}

[1] Adams, S., Boundary amenability for word hyperbolic groups and an application to smooth dynamics of simple groups, Topology, 33 (1994), 765-783.

[2] Anantharaman-Delaroche, C., Systmes dynamiques non commutatifs et moyennabilit, Math. Ann., 279 (1987), 297-315.

[3] Archbold, R. J. and Spielberg, J. S., Topologically free actions and ideals in discrete $C^{*}$-dynamical systems, Proc. Edinburgh Math. Soc. (2), 37 (1994), 119-124.

[4] Boyd, S., Keswani, N. and Raeburn, I., Faithful representations of crossed products by endomorphisms, Proc. Amer. Math. Soc., 118 (1993), 427-436.

[5] Choi, M. D., A simple $C^{*}$-algebra generated by two finite-order unitaries, Canad. J. Math., 31 (1979), 867-880.

[6] Cuntz, J., Simple $C^{*}$-algebras generated by isometries, Comm. Math. Phys., 57 (1977), $173-185$.

[7] - A class of $C^{*}$-algebras and topological Markov chains. II. Reducible chains and the Ext-functor for $C^{*}$-algebras, Invent. Math., 63 (1981), 25-40.

[8] Cuntz, J. and Krieger, W., A class of $C^{*}$-algebras and topological Markov chains, Invent. Math., 56 (1980), 251-268.

[9] Dykema, K. J., Exactness of reduced amalgamated free products of $C^{*}$-algebras, to appear in Proc. Edinburgh Math. Soc.

[10] $\longrightarrow$ Free products of exact groups. Preprint, 1999.

[11] Dykema, K. J. and Shlyakhtenko, D., Exactness of Cuntz-Pimsner $C^{*}$-algebras, to appear in Proc. Edinburgh Math. Soc.

[12] Enomoto, M., Fujii, M. and Watatani, Y., Tensor algebra on the sub-Fock space associated with $\mathcal{O}_{A}$, Math. Japon., 26 (1981), 171-177.

[13] - KMS states for gauge action on $O_{A}$, Math. Japon., 29 (1984), 607-619.

[14] Evans, D. E., Gauge actions on $\mathcal{O}_{A}$, J. Operator Theory, 7 (1982), 79-100.

[15] Figà-Talamanca, H. and Nebbia, C., Harmonic analysis and representation theory for groups acting on homogeneous trees, London Math. Soc. Lecture Note Ser., 162 (1991).

[16] Freudenthal, H., Über die Enden diskreter Räume und Gruppen, Comment. Math. Helv., 17 (1944), 1-38.

[17] Furstenberg, H., Boundary theory and stochastic processes on homogeneous spaces. Harmonic analysis on homogeneous spaces, Proc. Sympos. Pure Math., XXVI, Williams Coll., Williamstown, Mass., 1972, 193-229, Amer. Math. Soc., Providence, RI, 1973.

[18] Ghys, T. and de la Harpe, P., Sur les groupes hyperboliques d'aprs Mikhael Gromov, (Bern, 1988), Progr. Math., 83, Birkhuser Boston, Boston, MA, 1990.

[19] Kajiwara, T., Pinzari, C. and Watatani, Y., Ideal structure and simplicity of the $C^{*}$ algebras generated by Hilbert bimodules, J. Funct. Anal., 159 (1998), 295-322. 
[20] Kirchberg, E., On subalgebras of the CAR-algebra, J. Funct. Anal., 129 (1995), 35-63.

[21] Laca, M. and Spielberg, J., Purely infinite $C^{*}$-algebras from boundary actions of discrete groups, J. Reine Angew. Math., 480 (1996), 125-139.

[22] Pimsner, M. V., KK-groups of crossed products by groups acting on trees, Invent. Math., 86 (1986), 603-634.

[23] —, A class of $C^{*}$-algebras generalizing both Cuntz-Krieger algebras and crossed products by $Z$, Free probability theory, 189-212, Fields Inst. Commun., 12, Amer. Math. Soc., Providence, RI, 1997.

[24] Pimsner, M. and Voiculescu, D., Exact sequences for $K$-groups and Ext-groups of certain cross-product $C^{*}$-algebras, J. Operator Theory, 4 (1980), 93-118.

[25] Ramagge, J. and Robertson, G., Factors from trees, Proc. Amer. Math. Soc., 125 (1997), 2051-2055.

[26] Robertson, G. and Steger, T., $C^{*}$-algebras arising from group actions on the boundary of a triangle building, Proc. London Math. Soc., (3) 72 (1996), 613-637.

[27] Serre, J-P., Trees, Translated from the French by John Stillwell, Springer-Verlag, BerlinNew York, 1980.

[28] Spielberg, J., Free-product groups, Cuntz-Krieger algebras, and covariant maps, Internat. J. Math., 2 (1991), 457-476.

[29] Stacey, P. J., Crossed products of $C^{*}$-algebras by *-endomorphisms, J. Austral. Math. Soc. Ser. A, 54 (1993), 204-212.

[30] Szymański, W. and Zhang, S., Infinite simple $C^{*}$-algebras and reduced cross products of abelian $C^{*}$-algebras and free groups, Manuscripta Math., 92 (1997), 487-514.

[31] Wassermann, S., Exact $C^{*}$-algebras and related topics, Lecture Notes Ser., 19. Seoul National University, Research Institute of Mathematics, Global Analysis Research Center, Seoul, 1994.

[32] Woess, W., Boundaries of random walks on graphs and groups with infinitely many ends, Israel J. Math., 68 (1989), 271-301.

[33] - Random walks on infinite graphs and groups, Cambridge Tracts in Math., 138. Cambridge University Press, Cambridge, 2000. 